\title{
Pseudogroups associated with the one dimensional foliation group (II)
}

\author{
By Richard M. KOCH
}

(Received May 29, 1970)

\section{CHAPTER III: THE EQUIVALENCE PROBLEM AND THE PSEUDOGROUPS}

The purpose of this chapter is to complete the proof of the theorem below:

THEOREM 14. The following is a complete list of all pseudogroups associated with the one-dimensional foliation subgroup of $G L(N, R)$. (Note: We omit, throughout this list, the conditions $\frac{\partial f^{1}}{\partial x_{1}}>0$ and $\operatorname{det}\left(\frac{\partial f^{i}}{\partial x_{j}}\right)>0$, which guarantee that $\left(\frac{\partial f^{i}}{\partial x_{j}}\right)$ belongs to the connected group $G^{1}$.)

1.

$$
\left\{\begin{array}{l}
y_{1}=\sum_{j=1}^{N} a_{1 j} x_{j}+b_{1} \\
y_{i}=\sum_{j=2}^{N} a_{i j} x_{j}+b_{i}
\end{array}\right.
$$

2.

$$
\left\{\begin{array}{l}
y_{1}=c x_{1}+g\left(x_{2}, \cdots, x_{n}\right) \\
y_{i}=\sum_{j=2}^{N} a_{i j} x_{j}+b_{i}
\end{array}\right.
$$

$2 n$.

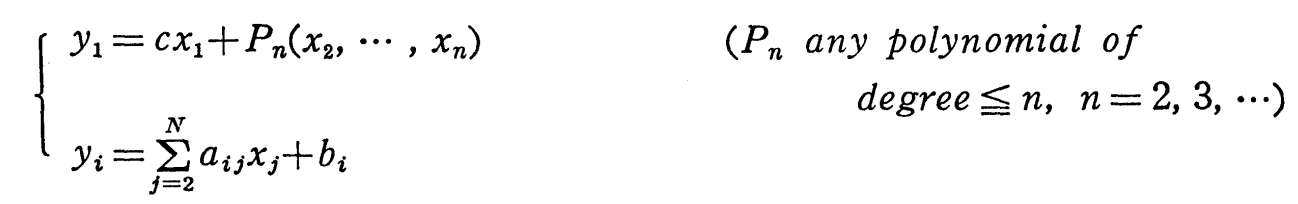

3.

$$
\left\{\begin{aligned}
y_{1} & =c x_{1}+g\left(x_{2}, \cdots, x_{n}\right) \\
y_{i} & =\frac{\sum_{j=2}^{N} a_{i j} x_{j}+b_{i}}{\sum_{j=2}^{N} c_{j} x_{j}+d}
\end{aligned}\right.
$$

4.

$$
\left\{\begin{array}{l}
y_{1}=c x_{1}+g\left(x_{2}, \cdots, x_{n}\right) \\
y_{i}=f_{i}\left(x_{2}, \cdots, x_{n}\right)
\end{array} \quad\left(\operatorname{det}\left(\frac{\partial f^{i}}{\partial x_{j}}\right)=\text { constant } ; N \geqq 3\right)\right.
$$

5.

$$
\left\{\begin{array}{l}
y_{1}=c x_{1}+g\left(x_{2}, \cdots, x_{n}\right) \\
y_{i}=f_{i}\left(x_{2}, \cdots, x_{n}\right)
\end{array}\right.
$$


6.

$$
\left\{\begin{array}{l}
y_{1}=x_{1} g\left(x_{2}, \cdots, x_{n}\right)+h\left(x_{2}, \cdots, x_{n}\right) \\
y_{i}=\sum_{j=2}^{N} a_{i j} x_{j}+b_{i}
\end{array}\right.
$$

$6 n$.

$$
\left\{\begin{array}{lr}
y_{1}=x_{1} e^{P_{n}\left(x_{2}, \cdots, x_{n}\right)}+h\left(x_{2}, \cdots, x_{n}\right) & \left(P_{n}\right. \text { any polynomial of } \\
y_{i}=\sum_{j=2}^{N} a_{i j} x_{j}+b_{i} & \text { degree } \leqq n, n=1,2, \cdots)
\end{array}\right.
$$

7.

$$
\left\{\begin{array}{l}
y_{1}=x_{1} g\left(x_{2}, \cdots, x_{n}\right)+h\left(x_{2}, \cdots, x_{n}\right) \\
y_{i}=\frac{\sum_{j=2}^{N} a_{i j} x_{j}+b_{i}}{\sum_{j=2}^{N} c_{i} x_{i}+d}
\end{array}\right.
$$

8.

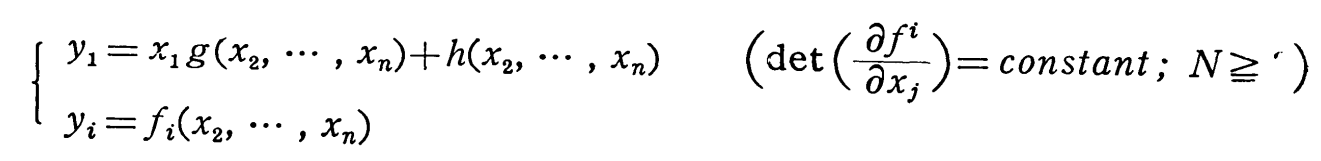

9.

$$
\left\{\begin{array}{l}
y_{1}=x_{1} g\left(x_{2}, \cdots, x_{n}\right)+h\left(x_{2}, \cdots, x_{n}\right) \\
y_{i}=f_{i}\left(x_{2}, \cdots, x_{n}\right)
\end{array}\right.
$$

$10 \lambda$.

$$
\left\{\begin{array}{l}
y_{1}=\frac{A x_{1}+h\left(x_{2}, \cdots, x_{n}\right)}{\left(\sum_{j=2}^{N} c_{j} x_{j}+d\right)^{\lambda}} \\
y_{i}=\frac{\sum_{j=2}^{N} a_{i j} x_{j}+b_{i}}{\sum_{j=2}^{N} c_{j} x_{j}+d}
\end{array} \quad(\lambda \text { fixed, } \lambda \neq 0)\right.
$$

$10 n$.

$$
\left\{\begin{array}{l}
y_{1}=\frac{A x_{1}+P_{n}\left(x_{2}, \cdots, x_{n}\right)}{\left(\sum_{j=2}^{N} c_{j} x_{j}+d\right)^{n}} \\
y_{i}=\frac{\sum_{j=2}^{N} a_{i j} x_{j}+b_{i}}{\sum_{j=2}^{N} c_{j} x_{j}+d}
\end{array}\right.
$$

$\left(P_{n}\right.$ any polynomial of degree $\leqq n, n=1,2, \cdots$ )

$11 \lambda$.

$$
\left\{\begin{array}{l}
y_{1}=C x_{1}\left[\operatorname{det}\left(\frac{\partial f^{i}}{\partial x_{j}}\right)\right]^{\lambda}+h\left(x_{2}, \cdots, x_{n}\right) \quad(\lambda \text { fixed }, \lambda \neq 0) \\
y_{i}=f_{i}\left(x_{2}, \cdots, x_{n}\right)
\end{array}\right.
$$

12.

$$
\left\{\begin{array}{l}
y_{1}=\frac{a\left(x_{2}, \cdots, x_{n}\right) x_{1}+b\left(x_{2}, \cdots, x_{n}\right)}{c\left(x_{2}, \cdots, x_{n}\right) x_{1}+d\left(x_{2}, \cdots, x_{n}\right)} \\
y_{i}=\sum_{j=2}^{N} a_{i j} x_{j}+b_{i}
\end{array}\right.
$$


13.

$$
\left\{\begin{array}{l}
y_{1}=\frac{a\left(x_{2}, \cdots, x_{n}\right) x_{1}+b\left(x_{2}, \cdots, x_{n}\right)}{c\left(x_{2}, \cdots, x_{n}\right) x_{1}+d\left(x_{2}, \cdots, x_{n}\right)} \\
y_{i}=\frac{\sum_{j=2}^{N} a_{i j} x_{j}+b_{i}}{\sum_{j=2}^{N} c_{j} x_{j}+d}
\end{array}\right.
$$

14.

$$
\left\{\begin{array}{l}
y_{1}=\frac{a\left(x_{2}, \cdots, x_{n}\right) x_{1}+b\left(x_{2}, \cdots, x_{n}\right)}{c\left(x_{2}, \cdots, x_{n}\right) x_{1}+d\left(x_{2}, \cdots, x_{n}\right)} \\
y_{i}=f_{i}\left(x_{2}, \cdots, x_{n}\right)
\end{array} \quad\left(\operatorname{det}\left(\frac{\partial f^{i}}{\partial x_{j}}\right)=\text { constant } ; N \geqq 3\right)\right.
$$

15. $\quad\left\{\begin{array}{l}y_{1}=\frac{a\left(x_{2}, \cdots, x_{n}\right) x_{1}+b\left(x_{2}, \cdots, x_{n}\right)}{c\left(x_{2}, \cdots, x_{n}\right) x_{1}+d\left(x_{2}, \cdots, x_{n}\right)} \\ y_{i}=f_{i}\left(x_{2}, \cdots, x_{n}\right)\end{array}\right.$

16.

$$
\left\{\begin{array}{l}
y_{1}=f_{1}\left(x_{1}, \cdots, x_{n}\right) \\
y_{i}=\sum_{j=2}^{N} a_{i j} x_{j}+b_{i}
\end{array}\right.
$$

17.

$$
\left\{\begin{array}{l}
y_{1}=f_{1}\left(x_{1}, \cdots, x_{n}\right) \\
y_{i}=\frac{\sum_{j=2}^{N} a_{i j} x_{j}+b_{i}}{\sum_{j=2}^{N} c_{j} x_{j}+d}
\end{array}\right.
$$

18.

$$
\left\{\begin{array}{l}
y_{1}=f_{1}\left(x_{1}, \cdots, x_{n}\right) \\
y_{i}=f_{i}\left(x_{2}, \cdots, x_{n}\right)
\end{array}\right.
$$

19.

$$
\left\{\begin{array}{l}
y_{1}=f_{1}\left(x_{1}, \cdots, x_{n}\right) \\
y_{i}=f_{i}\left(x_{2}, \cdots, x_{n}\right)
\end{array}\right.
$$

1. $G^{2}$

We will constantly refer to the previous list and to the list of kernels (the numbering on the two lists is different). In this section we will solve the equivalence problem for $G^{2}$ and determine the corresponding pseudogroups (which belong to $k^{2}, \Lambda^{3} k^{2}, \cdots$ ). In each case we must find $f$ such that $\theta^{2}(f)$ $-\varphi \in k^{2}$; then letting $\varphi=0$, we must find the most general solution to get the pseudogroup. As $\left(\frac{\partial f^{i}}{\partial x_{j}}\right) \in G^{1}, y_{1}=f_{1}\left(x_{1}, \cdots, x_{n}\right), y_{i}=f_{i}\left(x_{2}, \cdots, x_{n}\right) \quad(i \geqq 2)$. The equation to be solved can be written

$$
f_{j_{1} j_{2}}^{i}=\Sigma\left(\varphi_{k_{1} k_{2}}^{i}+k_{k_{1} k_{2}}^{i}\right) f^{k_{j_{1}}} f_{j_{j_{2}}}^{k_{k_{2}}}
$$

where $\varphi$ determines $g^{2}$ and so is fixed, but $k_{k_{1} k_{2}}^{i}$ may vary with $x_{1}, \cdots, x_{n}$, 
remaining in $k^{2}$.

Recall that $\varphi \in \Lambda^{2} k^{1} / k^{2}$.

LEMMA 47. Let $\xi_{j_{1} j_{2}}^{i}=\left(\begin{array}{ccc}e & f_{j} & g_{j_{1} j_{2}} \\ 0 & 0 & h^{i}{ }_{j_{1} j_{2}}\end{array}\right), \xi_{j}^{i}=\left(\begin{array}{ll}a & b_{i} \\ 0 & d^{i}{ }_{j}\end{array}\right)$. Then $\sum \xi_{k_{1} k_{2}}^{i} \xi_{j_{1}}^{k_{1}} \xi_{j_{2}}^{k_{2}}$

$$
=\left(\begin{array}{ccc}
e a^{2} & e a b_{j}+\sum f_{k} d_{j}^{k} a & \left(e b_{j_{1}} b_{j_{2}}+\sum f_{k} d_{j_{1}}^{k} b_{j_{2}}+\sum f_{k} d_{j_{2}}^{k} b_{j_{1}}+\sum g_{k_{1} k_{2}} d^{k_{j_{j_{1}}}} d^{k_{j_{2}}}{ }_{j_{2}}\right) \\
0 & 0 & h_{k_{1} k_{2}} d^{k_{j_{1}}} d^{k_{2}}{ }_{j_{2}}
\end{array}\right) .
$$

Proof. Calculate.

LEMMA 48. If $\xi^{i}{ }_{j_{1} j_{2}}=\left(\begin{array}{lll}e & f_{i} & g_{j_{1} j_{2}} \\ 0 & 0 & h^{i}{ }_{j_{1} j_{2}}\end{array}\right)$, the equations

$$
\frac{\partial^{2} \psi^{i}}{\partial x_{j_{1}} \partial x_{j_{2}}}=\Sigma \xi^{i}{ }_{k_{1} k_{2}} \frac{\partial \psi^{k_{1}}}{\partial x_{j_{1}}} \frac{\partial \psi^{k_{2}}}{\partial x_{j_{2}}}
$$

can be written explicitly as:

(A) $\frac{\partial^{2} \psi^{1}}{\partial x_{1}^{2}}=e\left(\frac{\partial \psi^{1}}{\partial x_{1}}\right)^{2}$

(B)

$$
\frac{\partial^{2} \psi^{1}}{\partial x_{1} \partial x_{j}}=e\left(\frac{\partial \psi^{1}}{\partial x_{1}}\right)\left(\frac{\partial \psi^{1}}{\partial x_{j}}\right)+\Sigma f_{k}\left(\frac{\partial \psi^{k}}{\partial x_{j}}\right)\left(\frac{\partial \psi^{1}}{\partial x_{1}}\right)
$$

(C)

$$
\begin{aligned}
& \frac{\partial^{2} \psi^{1}}{\partial x_{j_{1}} \partial x_{j_{2}}}=e\left(\frac{\partial \phi^{1}}{\partial x_{j_{1}}}\right)\left(\frac{\partial \psi^{1}}{\partial x_{j_{2}}}\right)+\sum f_{k}\left(\frac{\partial \phi^{k}}{\partial x_{j_{1}}}\right)\left(\frac{\partial \psi^{1}}{\partial x_{j_{2}}}\right) \\
& +\Sigma f_{k}\left(\frac{\partial \psi^{k}}{\partial x_{j_{2}}}\right)\left(\frac{\partial \psi^{1}}{\partial x_{j_{1}}}\right)+\Sigma g_{k_{1} k_{2}}\left(\frac{\partial \psi^{k_{1}}}{\partial x_{j_{1}}}\right)\left(\frac{\partial \psi^{k_{2}}}{\partial x_{j_{2}}}\right)
\end{aligned}
$$

(D) $\quad \frac{\partial^{2} \psi^{i}}{\partial x_{j_{1}} \partial x_{j_{2}}}=\Sigma h_{k_{1} k_{2}}^{i}\left(\frac{\partial \psi^{k_{1}}}{\partial x_{j_{1}}}\right)\left(\frac{\partial \phi^{k_{2}}}{\partial x_{j_{2}}}\right)$.

PROOF. A trivial consequence of Lemma 47.

LEMMA 49. If $\left(\begin{array}{lll}0 & 0 & 0 \\ 0 & 0 & T^{2}\end{array}\right) \in k^{2}$, (D) is irrelevant. If $\left(\begin{array}{lll}0 & 0 & g_{i j} \\ 0 & 0 & 0\end{array}\right) \in k^{2},(\mathrm{C})$ is irrelevant. If $\left(\begin{array}{ccc}0 & f_{i} & 0 \\ 0 & 0 & 0\end{array}\right) \in k^{2},(\mathrm{~B})$ is irrelevant.

Proof. For example, if the second condition holds, we can choose $g_{i j}\left(x_{1}, \cdots, x_{n}\right)$ so that (C) is satisfied, since $\frac{\partial \psi^{i}}{\partial x_{j}}$ is non-singular. The othe, cases follow in the same way.

LEMMA 50. The most general solution of (A), if $e=0$, is $x_{1} g\left(x_{2}, \cdots, x_{n}\right)$. $+h\left(x_{2}, \cdots, x_{n}\right)$. The most general solution of $(\mathrm{A})$, if $e$ is a non-zero constant, is $-\frac{1}{e} \ln \left(x_{1}+g\left(x_{2}, \cdots, x_{n}\right)\right)+h\left(x_{2}, \cdots, x_{n}\right)$.

Proof. Obvious.

We now discuss the list of kernels case by case, proceeding from the easier to the more difficult examples. Notice that (13) and (16) on the list of kernels are done, since $\Lambda^{2} k^{1} / k^{2}=0$. These obviously give rise to pseudogroups 
(19) on the list of pseudogroups.

Now turn to (8) and (11) on the kernel list. We can pick $\varphi=\left(\begin{array}{lll}e & 0 & 0 \\ 0 & 0 & 0\end{array}\right)$ and $k^{2}=\left(\begin{array}{ccc}0 & f_{j} & g_{j k} \\ 0 & 0 & h^{i}{ }_{j k}\end{array}\right)$. Consider the equivalence problem; lemmas 49 and 50 show that $\phi^{1}=-\frac{1}{e} \ln \left(x_{1}+1\right), \psi^{i}=x_{i}$ is a solution. Notice that $\phi$ is defined in a neighborhood of the origin and is a local diffeomorphism there $; \phi(0)=0$.

To find the pseudogroups, suppose $e=0$. Then $\phi^{1}=x_{1} g\left(x_{2}, \cdots, x_{n}\right)$ $+h\left(x_{2}, \cdots, x_{n}\right)$ by lemma 50 . This is example (9) on our list of pseudogroups.

Next turn to (3), (6), (17), and (18) and (18b) in the list of kernels. Each of these contains $\left(\begin{array}{lll}0 & 0 & g_{j k} \\ 0 & 0 & 0\end{array}\right) \in k^{2}$, so equation $(\mathrm{C})$ is irrelevant. In each case we can pick $\varphi=\left(\begin{array}{ccc}e & f_{i} & 0 \\ 0 & 0 & 0\end{array}\right)$. For the purpose of the equivalence problem consider $k_{j_{1} j_{2}}^{i}=\left(\begin{array}{lll}0 & 0 & e_{j_{1} j_{2}} \\ 0 & 0 & 0\end{array}\right)$ and let $\psi^{i}=x_{i}$. Then only

need be solved.

$$
\begin{aligned}
& \frac{\partial^{2} \psi^{1}}{\partial x_{1}{ }^{2}}=e\left(\frac{\partial \psi^{1}}{\partial x_{1}}\right)^{2} \\
& \frac{\partial^{2} \psi^{1}}{\partial x_{j} \partial x_{1}}=e\left(\frac{\partial \psi^{1}}{\partial x_{j}}\right)\left(\frac{\partial \phi^{1}}{\partial x_{1}}\right)+f_{j} \frac{\partial \psi^{1}}{\partial x_{1}}
\end{aligned}
$$

But if $e=0, \psi^{1}=x_{1} l_{1}\left(x_{2}, \cdots, x_{n}\right)+l_{2}\left(x_{2}, \cdots, x_{n}\right)$ and we have only to solve $\frac{\partial l_{1}}{\partial x_{j}}=f_{j} l_{1}$. If $l_{1}=e^{s\left(x_{2}, \cdots, x_{n}\right)}$, this becomes $\frac{\partial s}{\partial x_{j}}=f_{j}$ and as the $f_{j}$ are constants, this can be solved. If $e \neq 0$, we can pick $\phi^{1}=-\frac{1}{e} \ln \left(x_{1}+1\right)+l\left(x_{2}, \cdots, x_{n}\right)$. Then we need only solve

$$
0=e\left(-\frac{1}{e} \frac{1}{x+1}\right)\left(\frac{\partial l}{\partial x_{j}}\right)+f_{j}\left(-\frac{1}{e} \frac{1}{x+1}\right)
$$

or $\frac{\partial l}{\partial x_{j}}=-\frac{1}{e} f_{j}$ which again is easily done.

Next we determine the pseudogroups. Examine (3) and (6) first. By lemma 49, equations (C) and (D) are irrelevant. Hence

$$
\frac{\partial^{2} \psi^{1}}{\partial x_{1}^{2}}=0 \quad \frac{\partial^{2} \psi^{1}}{\partial x_{1} \partial x_{j}}=0
$$

Therefore $\phi^{1}=c x_{1}+g\left(x_{2}, \cdots, x_{n}\right), \psi^{i}=f_{i}\left(x_{2}, \cdots, x_{n}\right)$, which is (5) on the list of pseudogroups. In (17) and (18) we must solve

$$
\begin{aligned}
& \frac{\partial^{2} \psi^{1}}{\partial x_{1}{ }^{2}}=0 \\
& \frac{\partial^{2} \psi^{1}}{\partial x_{1} \partial x_{j}}=\sum \lambda d_{k k}^{(2)} \frac{\partial \psi^{1}}{\partial x_{1}} \frac{\partial \psi^{k}}{\partial x_{j}}
\end{aligned}
$$




$$
\frac{\partial^{2} \psi^{i}}{\partial x_{j_{1}} \partial x_{j_{2}}}=\Sigma d_{k_{1} k_{2}}^{i} \frac{\partial \phi^{k_{1}}}{\partial x_{j_{1}}} \frac{\partial \phi^{k_{2}}}{\partial x_{j_{2}}}
$$

with $d^{i}{ }_{j_{1} j_{2}}$ functions. Hence $\psi^{1}=x_{1} l_{1}\left(x_{2}, \cdots, x_{n}\right)+l_{2}\left(x_{2}, \cdots, x_{n}\right), \psi^{i}=f_{i}\left(x_{2}, \cdots, x_{n}\right)$ and

$$
\frac{\partial l_{1}}{\partial x_{j}}=\sum \lambda d_{k k}^{(2) k} \frac{\partial f^{k}}{\partial x_{j}} l_{1}
$$

Now $l_{1} \neq 0$ since $\frac{\partial \psi^{1}}{\partial x_{1}} \neq 0$, so $l_{1}=e^{s\left(x_{2}, \cdots, x_{n}\right)}$, at least locally, and

$$
\frac{\partial s}{\partial x_{j}}=\sum \lambda d^{(2) k}{ }_{k k} \frac{\partial f^{k}}{\partial x_{j}} \text {. }
$$

LEMMA 51. Suppose $\left\{f^{i}\left(x_{2}, \cdots, x_{N}\right)\right\}$ satisfy

Then

$$
\frac{\partial^{2} f^{i}}{\partial x_{j_{1}} \partial x_{j_{2}}}=\Sigma d_{k_{1} k_{2}}^{i} \frac{\partial f^{k_{1}}}{\partial x_{j_{1}}} \frac{\partial f^{k_{2}}}{\partial x_{j_{2}}} .
$$

$$
\Sigma d_{k k}^{(2) k} \frac{\partial f^{k}}{\partial x_{j}}=\frac{2}{N} \frac{\partial}{\partial x_{j}} \ln \operatorname{det}\left(\frac{\partial f^{i}}{\partial x_{j}}\right) .
$$

Proof. The identity

$$
\frac{\partial}{\partial x_{j}} \operatorname{det}\left(\frac{\partial f^{i}}{\partial x_{j}}\right)=\operatorname{det}\left(\frac{\partial f^{i}}{\partial x_{j}}\right) \sum \frac{\partial^{2} f^{k}}{\partial x_{r} \partial x_{j}}\left[\left(\frac{\partial f^{i}}{\partial x_{j}}\right)^{-1}\right]_{k}^{r}
$$

is well known. Applying the formula for $\frac{\partial^{2} f^{k}}{\partial x_{r} \partial x_{j}}$, we have

Thus

$$
\begin{aligned}
\frac{\partial}{\partial x_{j}} \operatorname{det}\left(\frac{\partial f^{i}}{\partial x_{j}}\right) & =\operatorname{det}\left(\frac{\partial f^{i}}{\partial x_{j}}\right) \Sigma h^{k}{ }_{s t} \frac{\partial f^{s}}{\partial x_{r}} \frac{\partial f^{t}}{\partial x_{j}}\left[\left(\frac{\partial f}{\partial x}\right)^{-1}\right]_{k}^{r} \\
& =\operatorname{det}\left(\frac{\partial f^{i}}{\partial x_{j}}\right) \Sigma h^{k}{ }_{k r} \frac{\partial f^{r}}{\partial x_{j}}
\end{aligned}
$$

$$
\begin{aligned}
\frac{\frac{\partial}{\partial x_{j}} \operatorname{det}\left(\frac{\partial f^{i}}{\partial x_{k}}\right)}{\operatorname{det}\left(\frac{\partial f^{i}}{\partial x_{k}}\right)} & =\frac{\partial}{\partial x_{j}} \ln \operatorname{det}\left(\frac{\partial f^{i}}{\partial x_{j}}\right) \\
& =\sum\left[\sum h^{(1) k}{ }_{k r}+\sum h^{(2) k}{ }_{k r}\right] \frac{\partial f^{r}}{\partial x_{j}}
\end{aligned}
$$

where $h=h^{(1)}+h^{(2)}, h^{(1)} \in(1-\Omega)\left(T^{2}\right), h^{(2)} \in \Omega\left(T^{2}\right)$. But then $\sum h^{(1) k}{ }_{k r}=0, h^{(2) k}{ }_{k r}$ $=\frac{1}{2}\left[\delta^{k}{ }_{k} h_{r r}^{(2) r}+\delta^{k}{ }_{r} h_{k k}^{(2) k}\right]$, so we have

$$
\begin{aligned}
\frac{1}{2}((N-1)+1) \sum h_{r r}^{(2) r} \frac{\partial f^{r}}{\partial x_{j}} & =\frac{N}{2} \sum h_{r r}^{(2) r} \frac{\partial f^{r}}{\partial x_{j}} \\
& =\frac{\partial}{\partial x_{j}} \ln \operatorname{det}\left(\frac{\partial f^{i}}{\partial x_{j}}\right) .
\end{aligned}
$$


Hence $\frac{\partial s}{\partial x_{j}}=\frac{\partial}{\partial x_{j}}\left[\frac{2 \lambda}{N} \ln \operatorname{det} \frac{\partial f^{i}}{\partial x_{j}}\right]$, so $\psi^{1}=C x_{1} \operatorname{det}\left(\frac{\partial f^{i}}{\partial x_{j}}\right)^{\frac{2 \lambda}{N}}+h\left(x_{2}, \cdots, x_{n}\right)$. Of course we can let $\tilde{\lambda}=\frac{2 \lambda}{N}$; then we obtain (11) on the list of pseudogroups.

Consider next (7), (9), (10), (12), (14), (15) on the kernel list; in each of these $\left(\begin{array}{ccc}0 & f_{i} & g_{i j} \\ 0 & 0 & 0\end{array}\right) \in k^{2}$, so by lemma 49, equations (B) and (C) are irrelevant. Moreover, $\varphi=\left(\begin{array}{lll}e & 0 & 0 \\ 0 & 0 & h^{i}{ }_{j k}\end{array}\right)$. In each case, $k^{2}$ can be taken in the form $\left(\begin{array}{ccc}0 & f_{i} & g_{j_{1} j_{2}} \\ 0 & 0 & k_{j_{1} j_{2}}^{i}\end{array}\right)$, with $k_{j_{1} j_{2}}^{i} \in \Omega\left(T^{2}\right),(1-\Omega)\left(T^{2}\right)$, or $T^{2}$, depending on the particular example considered. We must solve

$$
\begin{aligned}
& \frac{\partial^{2} \psi^{1}}{\partial x_{1}{ }^{2}}=e\left(\frac{\partial \psi^{1}}{\partial x_{1}}\right)^{2} \\
& \frac{\partial^{2} \psi^{i}}{\partial x_{j_{1}} \partial x_{j_{2}}}=\Sigma\left(h_{k_{1} k_{2}}+k_{k_{1} k_{2}}^{i}\right) \frac{\partial \psi^{k_{1}}}{\partial x_{j_{1}}} \frac{\partial \psi^{k_{2}}}{\partial x_{j_{2}}} .
\end{aligned}
$$

But the first can always be solved, and the second can be solved by theorem 11 . (To apply theorem 11, we need to know the required integrability conditions. Thus there must exist $\varphi^{i}{ }_{j k r}$ such that $\left\langle 1, \varphi^{i}{ }_{j k}, \varphi^{i}{ }_{j k r}\right\rangle$ is in $\Lambda^{3} g^{2}$. But it is easy to check that if this holds in the present $N$ dimensional case, it also holds in the lower $N-1$ dimensional block.)

Next we compute the pseudogroups. Consider $k^{2}=\left(\begin{array}{lll}e & e_{i} & e_{i j} \\ 0 & 0 & k^{i} j_{j_{1}}\end{array}\right)$; since (B) and (C) are still irrelevant we must solve

$$
\begin{aligned}
& \frac{\partial^{2} \psi^{1}}{\partial x_{1}{ }^{2}}=e\left(\frac{\partial \psi^{1}}{\partial x_{1}}\right)^{2} \\
& \frac{\partial^{2} \psi^{i}}{\partial x_{j_{1}} \partial x_{j_{2}}}=\Sigma k_{k_{1} k_{2}}^{i} \frac{\partial \psi^{k_{1}}}{\partial x_{j_{1}}} \frac{\partial \phi^{k_{2}}}{\partial x_{j_{2}}} .
\end{aligned}
$$

As $k^{2}$ varies over the several cases, either $e=0$ or $e$ is arbitrary. If $e=0$, $\psi^{1}=x_{1} g\left(x_{2}, \cdots, x_{n}\right)+h\left(x_{2}, \cdots, x_{n}\right)$; if $e$ is arbitrary, $\psi^{1}=f_{1}\left(x_{1}, \cdots, x_{n}\right)$. Moreover, $k^{i}{ }_{j k}$ varies over $\Omega\left(T^{2}\right),(1-\Omega)\left(T^{2}\right)$ and $T^{2}$, and thus generates one of the pseudogroups of theorem 12 . We get, then, (6), (7), (8), (16), (17), and (18) on the pseudogroup list.

Next consider (2), (4), and (5) in the list of kernels. Each $k^{2}$ contains $\left(\begin{array}{lll}0 & 0 & g_{i j} \\ 0 & 0 & 0\end{array}\right)$, so by lemma 49 equation (C) is irrelevant.

Letting $\varphi=\left(\begin{array}{lll}e & f_{i} & 0 \\ 0 & 0 & h^{i}{ }_{j k}\end{array}\right)$ and $k^{2}=\left(\begin{array}{lll}0 & 0 & g_{j k} \\ 0 & 0 & k^{i}{ }_{j_{1} j_{2}}\end{array}\right)$ we must solve

$$
\begin{aligned}
& \frac{\partial^{2} \psi^{1}}{\partial x_{1}{ }^{2}}=e\left(\frac{\partial \psi^{1}}{\partial x_{1}}\right)^{2} \\
& \frac{\partial^{2} \psi^{1}}{\partial x_{1} \partial x_{j_{1}}}=e \frac{\partial \psi^{1}}{\partial x_{1}} \frac{\partial \psi^{1}}{\partial x_{j_{1}}}+\Sigma f_{k} \frac{\partial \psi^{1}}{\partial x_{1}} \frac{\partial \psi^{k}}{\partial x_{j_{1}}}
\end{aligned}
$$




$$
\frac{\partial^{2} \psi^{i}}{\partial x_{j_{1}} \partial x_{j_{2}}}=\Sigma\left(h_{k_{1} k_{2}}^{i}+k_{k_{1} k_{2}}^{i}\right) \frac{\partial \psi^{k_{1}}}{\partial x_{j_{1}}} \frac{\partial \psi^{k_{2}}}{\partial x_{j_{2}}}
$$

As before, $\Lambda^{3} g^{2} \rightarrow g^{2}$ onto implies the required integrability condition for $h^{i}{ }_{k_{1} k_{2}}$, so the last equation can be solved.

If $e=0, \psi^{1}=x_{1} l_{1}\left(x_{2}, \cdots, x_{n}\right)+l_{2}\left(x_{2}, \cdots, x_{n}\right)$ and we must only solve $\frac{\partial l_{1}}{\partial x_{j_{1}}}$ $=\sum f_{k} \frac{\partial \psi^{k}}{\partial x_{j_{1}}} l_{1}$. But $l_{1} \neq 0$, so locally $l_{1}=e^{s\left(x_{2}, \cdots, x_{n}\right)}$; thus $\frac{\partial s}{\partial x_{j}}=\sum f_{k} \frac{\partial \psi^{k}}{\partial x_{j}}$. The integrability conditions for this equation are just

$$
\frac{\partial}{\partial x_{r}}\left[\Sigma f_{k} \frac{\partial \psi^{k}}{\partial x_{j}}\right]=\frac{\partial}{\partial x_{j}}\left[\Sigma f_{k} \frac{\partial \psi^{k}}{\partial x_{r}}\right]
$$

which hold because the $f_{k}$ are constant.

Now say $e \neq 0$. Then $\phi^{1}=-\frac{1}{e} \ln \left(x_{1}+1\right) l\left(x_{2}, \cdots, x_{n}\right)$ and we need only solve

$$
0=e\left(-\frac{1}{e\left(x_{1}+1\right)}\right) \frac{\partial l}{\partial x_{j}}+\Sigma f_{k} \frac{\partial \psi^{k}}{\partial x_{j}}\left(-\frac{1}{e\left(x_{1}+1\right)}\right)
$$

or $\frac{\partial l}{\partial x_{j}}=-\frac{1}{e} \sum f_{k} \frac{\partial \psi^{k}}{\partial x_{j}}$ which again can be done.

To find the pseudogroups, we solve

$$
\begin{aligned}
& \frac{\partial^{2} \psi^{1}}{\partial x_{1}{ }^{2}}=0 \\
& \frac{\partial^{2} \psi^{1}}{\partial x_{1} \partial x_{j}}=0 \\
& \frac{\partial^{2} \psi^{i}}{\partial x_{j_{1}} \partial x_{j_{2}}}=\sum k_{k_{1} k_{2}}^{i} \frac{\partial \psi^{k_{1}}}{\partial x_{j_{1}}} \frac{\partial \psi^{k_{2}}}{\partial x_{j_{2}}}
\end{aligned}
$$

Here $k_{k_{1} k_{2}}^{i} \in \Omega\left(T^{2}\right),(1-\Omega)\left(T^{2}\right)$, or $T^{2}$. But then $\phi^{1}=C x_{1}+h\left(x_{2}, \cdots, x_{n}\right)$ and $\psi^{i}$ belongs to one of the pseudogroups of theorem 12 , so $w$, have (2), (3), and (4) of the list of pseudogroups.

Consider (19) and (19b) in the list of kernels. $\left(\begin{array}{lll}0 & 0 & g_{j k} \\ 0 & 0 & 0\end{array}\right) \in k^{2}$, so equation (C) is irrelevant. We have

$$
\varphi=\left(\begin{array}{lll}
e & f_{i} & 0 \\
0 & 0 & k^{i}{ }_{r s}
\end{array}\right), \quad k_{j k}^{i}=\left(\begin{array}{ccc}
0 & \lambda d^{j}{ }_{j j} & g_{j k} \\
0 & 0 & d^{i}{ }_{j k}
\end{array}\right)
$$

and so must solve

$$
\begin{aligned}
& \frac{\partial^{2} \psi^{1}}{\partial x_{1}^{2}}=e\left(\frac{\partial \psi^{1}}{\partial x_{1}}\right)^{2} \\
& \frac{\partial^{2} \psi^{1}}{\partial x_{1} \partial x_{j_{1}}}=e-\frac{\partial \psi^{1}}{\partial x_{1}} \frac{\partial \psi^{1}}{\partial x_{j_{1}}}+\Sigma\left(f_{k}+\lambda d_{k k}^{k}\right) \frac{\partial \psi^{1}}{\partial x_{1}} \frac{\partial \psi^{k}}{\partial x_{j_{1}}} \\
& \frac{\partial^{2} \psi^{i}}{\partial x_{j_{1}} \partial x_{j_{2}}}=\Sigma\left(h^{i}{ }_{k_{1} k_{2}}+d_{k_{1} k_{2}}^{i}\right) \frac{\partial \psi^{k_{1}}}{\partial x_{j_{1}}} \frac{\partial \psi^{k_{2}}}{\partial x_{j_{2}}} \text {. }
\end{aligned}
$$


But these are precisely the equations we considered above with the addition of $\lambda d^{k}{ }_{k k}$, so we are done if

$$
\frac{\partial}{\partial x_{j}}\left[\Sigma\left(f_{k}+\lambda d_{k k}^{k}\right) \frac{\partial \phi^{k}}{\partial x_{r}}\right]=\frac{\partial}{\partial x_{r}}\left[\Sigma\left(f_{k}+\lambda d_{k k}^{k}\right) \frac{\partial \psi^{k}}{\partial x_{j}}\right]
$$

or, as $f_{k}$ is constant,

$$
\frac{\partial}{\partial x_{j}}\left[\Sigma d_{k k}^{k} \frac{\partial \psi^{k}}{\partial x_{r}}\right]=\frac{\partial}{\partial x_{r}}\left[\Sigma d_{k k}^{k} \frac{\partial \psi^{k}}{\partial x_{j}}\right] .
$$

By lemma 51,

$$
\Sigma\left(\varphi_{k k}^{(2) k}+d_{k k}^{(2) k}\right) \frac{\partial \psi^{k}}{\partial x_{j}}=\frac{2}{N} \frac{\partial}{\partial x_{j}} \ln \operatorname{det}\left(\frac{\partial \phi^{i}}{\partial x_{j}}\right) .
$$

In this case $d \in \Omega\left(T^{2}\right), \varphi \in(1-\Omega)\left(T^{2}\right)$, so $d^{(2)}=d, \varphi^{(2)}=0$, and we must have

$$
\frac{\partial}{\partial x_{j}}\left[\frac{2}{N}-\frac{\partial}{\partial x_{r}} \ln \operatorname{det}\left(\frac{\partial \psi^{i}}{\partial x_{j}}\right)\right]=\frac{\partial}{\partial x_{r}}\left[\frac{2}{N} \frac{\partial}{\partial x_{j}} \ln \operatorname{det}\left(\frac{\partial \psi^{i}}{\partial x_{j}}\right)\right]
$$

which, of course, holds.

To find the pseudogroup, we put $e, f_{i}, \varphi_{r s}^{i}=0$. Thus

$$
\begin{aligned}
& \frac{\partial^{2} \psi^{1}}{\partial x_{1}{ }^{2}}=0 \\
& \frac{\partial^{2} \psi^{1}}{\partial x_{1} \partial x_{j}}=\lambda \frac{2}{N} \frac{\partial}{\partial x_{j}}\left[\ln \operatorname{det}\left(\frac{\partial \psi^{i}}{\partial x_{j}}\right)\right] \frac{\partial \psi^{1}}{\partial x_{1}} \\
& \frac{\partial^{2} \psi^{1}}{\partial x_{j_{1}} \partial x_{j_{2}}}=\Sigma d_{k_{1} k_{2}}^{i} \frac{\partial \psi^{k_{1}}}{\partial x_{j_{1}}} \frac{\partial \psi^{k_{2}}}{\partial x_{j_{2}}} .
\end{aligned}
$$

Then $\psi^{1}=x_{1} e^{s\left(x_{2}, \cdots, x_{n}\right)}+l_{2}\left(x_{2}, \cdots, x_{n}\right)$

$$
\frac{\partial s}{\partial x_{j}}=\frac{2 \lambda}{N} \frac{\partial}{\partial x_{j}}\left[\ln \operatorname{det}\left(\frac{\partial \psi^{i}}{\partial x_{j}}\right)\right]
$$

and $\psi^{i}$ belongs to the projective pseudogroup $\psi^{i}=-\frac{\sum a_{i j} x_{j}+b_{i}}{\sum c_{j} x_{j}+d}$. We have

$$
\begin{aligned}
\psi^{1} & =C x_{1}\left(\operatorname{det} \frac{\partial \psi^{i}}{\partial x_{j}}\right)^{\frac{2 \lambda}{N}}+h\left(x_{2}, \cdots, x_{n}\right) \\
\psi^{i} & =\frac{\sum a_{i j} x_{j}+b_{i}}{\sum c_{j} x_{j}+d} ;
\end{aligned}
$$

since $\operatorname{det} \frac{\partial \psi^{i}}{\partial x_{j}} \neq 0$, this is (10) on the list of pseudogroups, provided:

LEMMA 52.

$$
\operatorname{det} \frac{\partial\left(\frac{\sum a_{i j} x_{j}+b_{i}}{\sum c_{j} x_{j}+d}\right)}{\partial x_{j}}=\frac{C}{\left(\sum c_{j} x_{j}+d\right)^{N}} \quad(C \text { a constant })
$$

PROOF. 


$$
\begin{aligned}
\frac{\partial \psi^{i}}{\partial x_{j}} & =\frac{\partial}{\partial x_{j}}\left(\frac{\sum a_{i j} x_{j}+b_{i}}{\sum c_{j} x_{j}+d}\right) \\
& =\frac{\left(\sum c_{k} x_{k}+d\right) a_{i j}-\left(\sum a_{i k} x_{k}+b_{i}\right) c_{j}}{\left(\sum c_{k} x_{k}+d\right)^{2}}=\frac{a_{i j}}{\sum c_{k} x_{k}+d}-\frac{c_{j}}{\sum c_{k} x_{k}+d} \psi^{i} \\
\frac{\partial^{2} \phi^{i}}{\partial x_{j_{1}} \partial x_{j_{2}}} & =-\frac{a_{i j_{1}} c_{j_{2}}}{\left(\sum c_{k} x_{k}+d\right)^{2}}+\frac{c_{j_{1}} c_{j_{2}}}{\left(\sum c_{k} x_{k}+d\right)^{2}} \psi^{i}-\frac{c_{j_{1}}}{\sum c_{k} x_{k}+d} \frac{\partial \psi^{i}}{\partial x_{j_{2}}} \\
& =\frac{\partial \psi^{i}}{\partial x_{j_{1}}}\left(\frac{-c_{j_{2}}}{\sum c_{k} x_{k}+d}\right)+\frac{\partial \phi^{i}}{\partial x_{j_{2}}}\left(\frac{-c_{j_{1}}}{\sum c_{k} x_{k}+d}\right) .
\end{aligned}
$$

But also,

$$
\begin{aligned}
\frac{\partial^{2} \psi^{i}}{\partial x_{j_{1}} \partial x_{j_{2}}} & =\sum h_{k_{1} k_{2}}^{i}-\frac{\partial \psi^{k_{1}}}{\partial x_{j_{1}}} \frac{\partial \psi^{k_{2}}}{\partial x_{j_{2}}} \\
& =\frac{1}{2}\left[\delta_{k_{1}}^{i} h^{\left.k_{2}{ }_{k_{2} k_{2}}+\delta^{i}{ }_{k_{2}} h^{k_{1}}{ }_{k_{1} k_{1}}\right]} \frac{\partial \psi^{k_{1}}}{\partial x_{j_{1}}} \frac{\partial \psi^{k_{2}}}{\partial x_{j_{2}}}\right. \\
& =\frac{\partial \psi^{i}}{\partial x_{j_{1}}} \frac{1}{N} \frac{\partial}{\partial x_{j_{2}}} \ln \operatorname{det}\left(\frac{\partial \psi^{i}}{\partial x_{j}}\right)+\frac{\partial \psi^{i}}{\partial x_{j_{2}}} \frac{1}{N} \frac{\partial}{\partial x_{j_{1}}} \ln \operatorname{det}\left(\frac{\partial \psi^{i}}{\partial x_{j}}\right) .
\end{aligned}
$$

Hence

$$
\begin{aligned}
0= & \frac{\partial \psi^{i}}{\partial x_{j_{1}}}\left[\frac{1}{N} \frac{\partial}{\partial x_{j_{2}}} \ln \operatorname{det}\left(\frac{\partial \psi^{i}}{\partial x_{j}}\right)+\frac{c_{j_{2}}}{\sum c_{k} x_{k}+d}\right] \\
& +\frac{\partial \psi^{i}}{\partial x_{j_{2}}}\left[\frac{1}{N} \frac{\partial}{\partial x_{j_{1}}} \ln \operatorname{det}\left(\frac{\partial \psi^{i}}{\partial x_{j}}\right)+\frac{c_{j_{1}}}{\sum c_{k} x_{k}+d}\right] .
\end{aligned}
$$

Since there is some $\langle i, j\rangle$ with $\frac{\partial \psi^{i}}{\partial x_{j}} \neq 0$, letting $j_{1}=j_{2}$ we get

$$
\frac{1}{N}-\frac{\partial}{\partial x_{j}} \ln \operatorname{det} \frac{\partial \phi^{i}}{\partial x_{j}}=-\frac{c_{j}}{\sum c_{k} x_{k}+d}=-\frac{\partial}{\partial x_{j}} \ln \left(\sum c_{k} x_{k}+d\right) \text {. }
$$

\section{Hence}

$$
-\frac{\partial}{\partial x_{j}}\left[\frac{1}{N} \ln \operatorname{det} \frac{\partial \psi^{i}}{\partial x_{j}}+\ln \left(\Sigma c_{k} x_{k}+d\right)\right]=0,
$$

so

$$
e^{\frac{1}{N} \ln \operatorname{det} \frac{\partial \psi_{i}^{i}}{\partial r_{j}} e^{l n\left(\Sigma \kappa_{k} \boldsymbol{k}^{+}+d\right)}}=D
$$

or

and we are done.

$$
\left(\operatorname{det} \frac{\partial \psi^{i}}{\partial x_{j}}\right)^{\frac{1}{N}}=\frac{D}{\sum c_{k} x_{k}+d}
$$

The final two examples force us to pay more attention to the requirement that $\Lambda^{3} g^{2} \rightarrow g^{2}$ be onto. Look at (1) in the list of kernels. Then $k^{2}=0$, and we are to solve the equations in lemma 48 with $\varphi=\left(\begin{array}{ccc}e & f_{i} & g_{i j} \\ 0 & 0 & h^{i}{ }_{j k}\end{array}\right)$. But since $\Lambda^{3} g^{2} \rightarrow g^{2}$ is onto, we can find $\varphi^{i}{ }_{j k r}$ so that $\left\langle 1, \varphi^{i}{ }_{j k}, \varphi^{i}{ }_{j k r}\right\rangle \in \Lambda^{3} g^{2}$. Then 


$$
\varphi^{i}{ }_{k j_{1} j_{2}}=\Sigma\left[\varphi_{k r}^{i} \varphi_{j_{1} j_{2}}-\varphi_{j_{1} r}^{i} \varphi^{r}{ }_{k j_{2}}-\varphi^{i}{ }_{j_{2} r} \varphi^{r}{ }_{k j_{1}}\right] .
$$

This must be symmetric in $k$ and $j_{1}$, so

$$
\sum \varphi_{j_{1} r}^{i} \varphi_{j_{2} j_{3}}^{r}=\sum \varphi_{j_{2} r}^{i} \varphi_{j_{1} j_{3}}^{r} .
$$

In particular if $j_{1}=1, j_{2}, j_{3} \geqq 2, i=1$, we have

$$
\varphi^{1}{ }_{11} \varphi_{j_{2} j_{3}}^{1}+\sum_{k \leq 2} \varphi^{1}{ }_{1 k} \varphi_{j_{2} j_{3}}^{k}=\varphi_{j_{2} 1}^{1} \varphi_{j_{3} 1}
$$

or

$$
e g_{j_{2} j_{3}}+\sum f_{k} h_{j_{2} j_{3}}^{k}=f_{j_{2}} f_{j_{3}}
$$

Similarly, $j_{1}, j_{2}, j_{3} \geqq 2, i=1$ implies

$$
f_{j_{1}} g_{j_{2} j_{3}}+\sum g_{k j_{1}} h_{j_{2} j_{3}}^{k}=f_{j_{2}} g_{j_{1} j_{3}}+\sum g_{k j_{2}} h_{j_{1} j_{3}}^{k}
$$

Let us now solve the equations of lemma 48. Clearly $\Lambda^{3} g^{2} \rightarrow g^{2}$ onto implies the usual integrability conditions on $h^{i}{ }_{j_{1} j_{2}}$, so (D) can be solved.

Let $e \neq 0$. Then $\phi^{1}=-\frac{1}{e} \ln \left(x_{1}+1\right)+l\left(x_{2}, \cdots, x_{n}\right)$ solves (A); (B) becomes

$$
0=\frac{-\frac{\partial l}{\partial x_{j}}}{x_{1}+1}+\Sigma f_{k} \frac{\partial \psi^{k}}{\partial x_{j}}\left(-\frac{1}{e\left(x_{1}+1\right)}\right)
$$

or $\frac{\partial l}{\partial x_{j}}=-\frac{1}{e} \sum f_{k} \frac{\partial \psi^{k}}{\partial x_{j}}$. Hence we can let $l=-\frac{1}{e} \sum f_{k} \psi^{k}$. Consider (C); it now reads

$$
\begin{aligned}
-\frac{1}{e} \Sigma f_{k} & \frac{\partial^{2} \psi^{k}}{\partial x_{j_{1}} \partial x_{j_{2}}} \\
= & \frac{1}{e} \sum f_{k_{1}} f_{k_{2}} \frac{\partial \psi^{k_{1}}}{\partial x_{j_{1}}} \frac{\partial \psi^{k_{2}}}{\partial x_{j_{2}}}+\sum f_{k_{1}} \frac{\partial \psi^{k_{1}}}{\partial x_{j_{1}}}\left[\frac{-1}{e} \Sigma f_{k_{2}} \frac{\partial \phi^{k_{2}}}{\partial x_{j_{2}}}\right] \\
& +\sum f_{k} \frac{\partial \phi^{k_{2}}}{\partial x_{j_{2}}}\left[\frac{-1}{e} \Sigma f_{k_{1}} \frac{\partial \phi^{k_{1}}}{\partial x_{j_{1}}}\right]+\Sigma g_{k_{1} k_{2}} \frac{\partial \psi^{k_{1}}}{\partial x_{j_{1}}}-\frac{\partial \psi^{k_{2}}}{\partial x_{j_{2}}}
\end{aligned}
$$

or

$$
\begin{aligned}
& \frac{-1}{e} \sum f_{k} h_{r_{1} r_{2}}^{k} \frac{\partial \psi^{r_{1}}}{\partial x_{j_{1}}} \frac{\partial \psi^{r_{2}}}{\partial x_{j_{2}}} \\
& \quad=\frac{-1}{e} \sum f_{r_{1}} f_{r_{2}} \frac{\partial \psi^{r_{1}}}{\partial x_{j_{1}}} \frac{\partial \psi^{r_{2}}}{\partial x_{j_{2}}}+\frac{1}{e^{2}} \sum g_{k_{1} k_{2}} f_{r_{1}}^{k_{1}} f_{r_{2}}^{k_{2}} \frac{\partial \psi^{r_{1}}}{\partial x_{j_{1}}} \frac{\partial \psi^{r_{2}}}{\partial x_{j_{2}}}
\end{aligned}
$$

Since $\frac{\partial^{2} \psi^{k}}{\partial x_{j_{1}} \partial x_{j_{2}}}=\sum h_{r_{1} r_{2}}^{k} \frac{\partial \psi^{r_{1}}}{\partial x_{j_{1}}} \frac{\partial \psi^{r_{2}}}{\partial x_{j_{2}}}$, this equation holds because $e g_{j_{2} j_{3}}+\sum f_{k} h_{j_{2} j_{3}}^{k}$ $=f_{j_{2}} f_{j_{3}}$.

Suppose $e=0$. Then $\psi^{1}=e^{s\left(x_{2}, \cdots, x_{n}\right)}\left(x_{1}+l\left(x_{2}, \cdots, x_{n}\right)\right)$ solves (A), and (B) 
reads $\frac{\partial s}{\partial x_{j}}=\sum f_{k} \frac{\partial \psi^{k}}{\partial x_{j}}$, so $s=\sum f_{k} \psi^{k}$. But

$$
\frac{\partial^{2} s}{\partial x_{j_{1}} \partial x_{j_{2}}}=\Sigma f_{k} h_{r_{1} r_{2}}^{k} \frac{\partial \psi^{r_{1}}}{\partial x_{j_{1}}} \frac{\partial \psi^{r_{2}}}{\partial x_{j_{2}}}=\Sigma f_{r_{1}} f_{r_{2}} \frac{\partial \psi^{r_{1}}}{\partial x_{j_{1}}} \frac{\partial \psi^{r_{2}}}{\partial x_{j_{2}}}=\frac{\partial s}{\partial x_{j_{1}}} \frac{\partial s}{\partial x_{j_{2}}}
$$

since $\sum f_{k} h^{k}{ }_{j_{1} j_{2}}=f_{j_{1}} f_{j_{2}}$ by the first boxed-in formula above.

Finally, (C) reads

$$
\begin{aligned}
\frac{\frac{\partial}{\partial x_{j_{1}}}}{2}\left[e^{s} \frac{\partial s}{\partial x_{j_{2}}}\left(x_{1}+l\right)+e^{s} \frac{\partial l}{\partial x_{j_{2}}}\right] & \\
= & e^{s}-\frac{\partial s}{\partial x_{j_{1}}} \frac{\partial s}{\partial x_{j_{2}}}\left(x_{1}+l\right)+e^{s} \frac{\partial^{2} s}{\partial x_{j_{1}} \partial x_{j_{2}}}\left(x_{1}+l\right)+e^{s} \frac{\partial s}{\partial x_{j_{1}}} \frac{\partial l}{\partial x_{j_{2}}} \\
& +e^{s} \frac{\partial s}{\partial x_{j_{2}}} \frac{\partial l}{\partial x_{j_{1}}}+e^{s} \frac{\partial^{2} l}{\partial x_{j_{1}} \partial x_{j_{2}}} \\
= & \frac{\partial s}{\partial x_{j_{1}}}\left[e^{s} \frac{\partial s}{\partial x_{j_{2}}}\left(x_{1}+l\right)+e^{s} \frac{\partial l}{\partial x_{j_{2}}}\right]+\frac{\partial s}{\partial x_{j_{2}}}\left[e^{s} \frac{\partial s}{\partial x_{j_{1}}}\left(x_{1}+l\right)+e^{s} \frac{\partial l}{\partial x_{j_{1}}}\right] \\
& +\sum g_{k_{1} k_{2}} \frac{\partial \psi^{k_{1}}}{\partial x_{j_{1}}} \frac{\partial \psi^{k_{2}}}{\partial x_{j_{2}}}
\end{aligned}
$$

or

$$
\frac{\partial^{2} l}{\partial x_{j_{1}} \partial x_{j_{2}}}=e^{-8} \Sigma g_{k_{1} k_{2}} \frac{\partial \psi^{k_{1}}}{\partial x_{j_{1}}} \frac{\partial \psi^{k_{2}}}{\partial x_{j_{2}}}
$$

Now in general $-\frac{\partial^{2} l}{\partial x_{j_{1}} \partial x_{j_{2}}}=R_{j_{1} j_{2}}$ can be solved if and only if $\frac{\partial R_{j_{1} j_{2}}}{\partial x_{j_{3}}}$ is a symmetric tensor. But

$$
\begin{aligned}
\frac{\partial}{\partial x_{j_{3}}}[ & \left.e^{-s} \sum g_{k_{1} k_{2}} \frac{\partial \psi^{k_{1}}}{\partial x_{j_{1}}} \frac{\partial \psi^{k_{2}}}{\partial x_{j_{2}}}\right] \\
= & e^{-s} \sum g_{k_{1} k_{2}} h^{k_{1}}{ }_{r_{1} r_{2}} \frac{\partial \psi^{r_{1}}}{\partial x_{j_{1}}} \frac{\partial \psi^{r_{2}}}{\partial x_{j_{2}}} \frac{\partial \psi^{k_{2}}}{\partial x_{j_{3}}}+e^{-s} \sum g_{k_{1} k_{2}} h_{r_{1} r_{2}}^{k_{2}} \frac{\partial \psi^{k_{1}}}{\partial x_{j_{1}}} \frac{\partial \psi^{r_{1}}}{\partial x_{j_{2}}} \frac{\partial \psi^{r_{2}}}{\partial x_{j_{3}}} \\
& -e^{-s} \sum f_{k_{1}} g_{k_{2} k_{3}-\frac{\partial \psi^{2}}{\partial x_{j_{1}}}} \frac{\partial \psi^{k_{3}}}{\partial x_{j_{2}}} \frac{\partial \psi^{k_{1}}}{\partial x_{j_{3}}} .
\end{aligned}
$$

Hence it suffices if $\Sigma\left[g_{j_{1} k} h_{j_{2} j_{3}}^{k}+g_{k_{2}} h^{k}{ }_{j_{1} j_{3}}\right]-f_{j_{3}} g_{j_{1} j_{2}}$ is symmetric, or subtracting $\mathcal{S} \sum g_{j_{1} k} h_{j_{2} j_{3}}^{k}$, if $f_{j_{3}} g_{j_{1} j_{2}}+\sum g_{k j_{3}} h_{j_{1} j_{2}}^{k}$ is symmetric, and this is the second boxed-in condition. The resulting pseudogroup obviously consists of all linear maps, (1) in our list.

Consider (19a) on the list of kernels. $\varphi=\left(\begin{array}{ccc}e & f_{i} & g_{i j} \\ 0 & 0 & h^{i}{ }_{j k}\end{array}\right), h^{i}{ }_{j k} \in(1-\Omega)\left(T^{2}\right)$ and

$$
k^{2}=\left(\begin{array}{ccc}
0 & \frac{1}{2} d_{j j}^{j} & 0 \\
0 & 0 & \Omega\left(T^{2}\right)
\end{array}\right) .
$$

Again, the equation for $\phi^{i}$ can be solved by theorem 11 . 
We now discuss the requirement that $\Lambda^{3} g^{2} \rightarrow g^{2}$ be onto. There must exist a $\varphi_{j k r}^{i}$ so $\left\langle 1, \varphi_{j k}^{i}, \varphi_{j k r}^{i}\right\rangle \in \Lambda^{3} g^{2}$; this means that for each fixed $j_{0}$, ther is ${ }^{j_{0}} k^{i}{ }_{j_{1} j_{2}}$ such that

$$
\varphi^{i}{ }_{j_{0} j_{1} j_{2}}=-\Sigma\left[\varphi_{j_{0} r}^{i} \varphi_{j_{1} j_{2}}-\varphi_{j_{1} r}^{i} \varphi_{j_{0} j_{2}}-\varphi_{j_{2} r}^{i} \varphi_{j_{0} j_{1}}\right]+{ }^{j_{0}} k_{j_{1} j_{2}}{ }^{i}
$$

But if $j_{0} \geqq 2, j_{1} \geqq 2, j_{2} \geqq 2, i=1,{ }^{j_{0}} k^{i}{ }_{j_{1} j_{2}}=0$ since

$$
k^{2}=\left(\begin{array}{ccc}
0 & \frac{1}{2} d^{j}{ }_{j j} & 0 \\
0 & 0 & d^{i}{ }_{j k}
\end{array}\right),
$$

so

$$
\Sigma\left[\varphi_{j_{0} r}^{1} \varphi_{j_{1} j_{2}}^{r}-\varphi^{1}{ }_{j_{1} r} \varphi^{r}{ }_{j_{0} j_{2}}-\varphi^{1}{ }_{j_{2} r} \varphi^{r}{ }_{j_{0} j_{1}}\right]
$$

must be symmetric in $j_{0}, j_{1}$; therefore $\sum \varphi^{1}{ }_{j_{0}} \varphi^{r}{ }_{j_{1} j_{2}}=\sum \varphi^{1}{ }_{j_{1} r} \varphi^{r}{ }_{j_{0} j_{2}}$, that is

$$
f_{j_{0}} g_{j_{1} j_{2}}+\sum g_{j_{0} k} h_{j_{1} j_{2}}^{k} \text { is symmetric. }
$$

But let $i=j_{2}=1, j_{0} \geqq 2, j_{1} \geqq 2$. Th $\mathrm{n}$

or

$$
\begin{aligned}
\varphi^{1}{ }_{j_{0} j_{1} 1} & =-\left[f_{j_{0}} f_{j_{1}}-f_{j_{1}} f_{j_{0}}-e g_{j_{0} j_{1}}-\sum f_{k} h^{k}{ }_{j_{0} j_{1}}\right]+{ }^{j_{0}} k^{i}{ }_{j_{1} 1} \\
\varphi^{1}{ }_{1 j_{0} j_{1}} & =-\left[e g_{j_{0} j_{1}}+\Sigma f_{k} h_{j_{0} j_{1}}^{k}-f_{j_{0}} f_{j_{1}}-f_{j_{1}} f_{j_{0}}\right]
\end{aligned}
$$

$$
e g_{j_{0} j_{1}}+\sum f_{k} h_{j_{0} j_{1}}-f_{j_{0} j_{1}}=-\frac{{ }^{j_{0} k^{j_{1}} j_{1} j_{1}}}{4} \text {. }
$$

Hence ${ }^{j_{0}} k^{j_{1}}{ }_{j_{1} j_{1}}={ }^{j_{1}} k^{j_{0}}{ }_{j_{0} j_{0}}$.

Looking at $i \geqq 2, j_{0} \geqq 2, j_{1} \geqq 2, j_{2} \geqq 2$, we see that

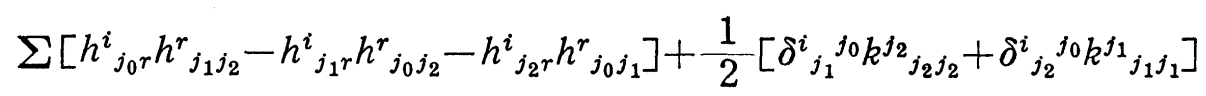

must be symmetric in $j_{0}, j_{1}$, and $j_{2}$. Thus interchanging $j_{0}$ and $j_{1}$ and subtracting,

$$
\sum 2\left(h_{j_{0} r}^{i} h_{j_{1} j_{2}}^{r}-h^{i}{ }_{j_{1} r} h^{r}{ }_{j_{0} j_{2}}\right)+\frac{1}{2}\left[\delta^{i}{ }_{j_{1}}{ }^{j_{0}} k^{j_{2}}{ }_{j_{2} j_{2}}-\delta^{i}{ }_{j_{0}}{ }^{j_{1}} k^{j_{2}}{ }_{j_{2} j_{2}}\right]=0 .
$$

If $j_{0} \neq j_{1}$, and $i=j_{0}$, we have

$$
\Sigma\left(h^{j_{0} j_{0} r} h_{j_{1} j_{2}}^{r}-h^{j_{0} j_{1} r} h_{j_{0} j_{2}}^{r}\right)=-\frac{1}{4}{ }^{j_{1}} k^{j_{2}}{ }_{j_{2} j_{2}} .
$$

If $j_{0}=j_{1}$, the left-hand side is zero. Summing over all $j_{0}$, we conclude, since $\varphi \in(1-\Omega)\left(T^{2}\right)$,

$$
-\frac{(N-2)}{4}{ }_{j_{1}} k_{j_{2} j_{2}}^{j_{2}}=-\sum h_{j_{0} r}^{j_{0}} h_{j_{0} j_{2}}^{r}
$$

Hence, since in (19a) $N>2$, we have 


$$
e g_{j_{0} j_{1}}+\sum f_{k} h_{j_{0} j_{1}}^{k}-f_{j_{0} j_{1}}=-\frac{1}{N-2} \sum h_{j_{0} k}^{r} h_{r j_{1}}^{k}
$$

These are sufficient conditions to solve our equations.

Now let $e \neq 0$. Let $\phi^{1}=-\frac{1}{e} \ln \left(x_{1}+1\right)+s\left(x_{2}, \cdots, x_{n}\right)$. Equation (B) becomes

$$
0=\frac{-\frac{\partial s}{\partial x_{j}}}{x_{1}+1}+\Sigma\left(f_{k}+\frac{1}{2} d_{k k}^{k}\right)\left(-\frac{1}{e\left(x_{1}+1\right)}\right) \frac{\partial \phi^{k}}{\partial x_{j}}
$$

or

$$
\begin{aligned}
\frac{\partial s}{\partial x_{j}} & =-\frac{1}{e} \Sigma\left(f_{k}+\frac{1}{2} d^{k}{ }_{k k}\right) \frac{\partial \psi^{k}}{\partial x_{j}} \\
& =-\frac{1}{e} \frac{\partial}{\partial x_{j}}\left[\Sigma f_{k} \psi^{k}+\frac{1}{N} \ln \operatorname{det} \frac{\partial \psi^{i}}{\partial x_{j}}\right] .
\end{aligned}
$$

Hence, let $s=-\frac{1}{e}\left(\sum f_{k} \psi^{k}+\frac{1}{N} \ln \operatorname{det} \frac{\partial \psi^{i}}{\partial x_{j}}\right)$. Equation (C) becomes (writing let $\left.\frac{\partial \psi^{i}}{\partial x_{j}}=\Delta\right)$ :

$$
\begin{aligned}
& \frac{\partial^{2}}{\partial x_{j_{1}} \partial x_{j_{2}}}\left[-\frac{1}{e}\left(\sum f_{k} \psi^{k}+\frac{1}{N} \ln \Delta\right)\right] \\
& =\frac{1}{e} \frac{\partial}{\partial x_{j_{1}}}\left[\Sigma f_{k} \psi^{k}+\frac{1}{N} \ln \Delta\right] \frac{\partial}{\partial x_{j_{2}}}\left[\Sigma f_{k} \psi^{k}+\frac{1}{N} \ln \Delta\right] \\
& \quad+\Sigma\left(f_{k}+\frac{1}{2} d^{k}{ }_{k k}\right) \frac{\partial \psi^{k}}{\partial x_{j_{1}}} \frac{\partial}{\partial x_{j_{2}}}\left[-\frac{1}{e}\left(\Sigma f_{k} \psi^{k}+\frac{1}{N} \ln \Delta\right)\right] \\
& \quad+\Sigma\left(f_{k}+\frac{1}{2}{d^{k}}_{k k}^{k}\right) \frac{\partial \psi^{k}}{\partial x_{j_{2}}} \frac{\partial}{\partial x_{j_{1}}}\left[-\frac{1}{e}\left(\Sigma f_{k} \psi^{k}+\frac{1}{N} \ln \Delta\right)\right] \\
& \quad+g_{k_{1} k_{2}} \frac{\partial \phi^{k_{1}}}{\partial x_{j_{1}}} \frac{\partial{\psi^{k}}^{k}}{\partial x_{j_{2}}}
\end{aligned}
$$

Hence it suffices if $l=\sum f_{k} \psi^{k}+\frac{1}{N} \ln \Delta$ and

But

$$
\frac{\partial^{2} l}{\partial x_{j_{1}} \partial x_{j_{2}}}=\frac{\partial l}{\partial x_{j_{1}}} \frac{\partial l}{\partial x_{j_{2}}}-\Sigma e g_{k_{1} k_{2}} \frac{\partial \psi^{k_{1}}}{\partial x_{j_{1}}} \frac{\partial \psi^{k_{2}}}{\partial x_{j_{2}}} \text {. }
$$

$$
\begin{aligned}
\frac{\partial^{2} l}{\partial x_{j_{1}} \partial x_{j_{2}}}= & \sum f_{k}\left(h^{k}{ }_{r_{1} r_{2}}+d^{k}{ }_{r_{1} r_{2}}\right) \frac{\partial \psi^{r_{1}}}{\partial x_{j_{1}}} \frac{\partial \psi^{r_{2}}}{\partial x_{j_{2}}}+\frac{1}{N} \frac{\partial^{2} \ln \Delta}{\partial x_{j_{1}} \partial x_{j_{2}}} \\
= & \sum f_{k} h^{k}{ }_{r_{1} r_{2}} \frac{\partial \psi^{r_{1}}}{\partial x_{j_{1}}} \frac{\partial \psi^{r_{2}}}{\partial x_{j_{2}}}+\sum f_{s} \frac{\partial \psi^{s}}{\partial x_{j_{1}}} \frac{1}{N} \frac{\partial}{\partial x_{j_{2}}} \ln \Delta \\
& +\sum f_{s} \frac{\partial \psi^{s}}{\partial x_{j_{2}}} \frac{1}{N} \frac{\partial}{\partial x_{j_{1}}} \ln \Delta+\frac{1}{N} \frac{\partial^{2}}{\partial x_{j_{1}} \partial x_{j_{2}}} \ln \Delta .
\end{aligned}
$$


so

$$
\frac{\partial l}{\partial x_{j_{1}}} \frac{\partial l}{\partial x_{j_{2}}}=\left[\sum f_{k} \frac{\partial \phi^{k}}{\partial x_{j_{1}}}+\frac{1}{N} \frac{\partial \ln \Delta}{\partial x_{j_{1}}}\right]\left[\sum f_{k} \frac{\partial \psi^{k}}{\partial x_{j_{2}}}+\frac{1}{N} \frac{\partial \ln \Delta}{\partial x_{j_{2}}}\right]
$$

However

$$
\begin{aligned}
\frac{\partial^{2} l}{\partial x_{j_{1}} \partial x_{j_{2}}}-\frac{\partial l}{\partial x_{j_{1}}} \frac{\partial l}{\partial x_{j_{2}}}= & \sum\left[\sum f_{k} h_{r_{1} r_{2}}^{k}-f_{r_{1}} f_{r_{2}}\right] \frac{\partial \phi^{r_{1}}}{\partial x_{j_{1}}} \frac{\partial \phi^{r_{2}}}{\partial x_{j_{2}}} \\
& +\frac{1}{N} \frac{\partial^{2} \ln \Delta}{\partial x_{j_{1}} \partial x_{j_{2}}}-\frac{1}{N^{2}} \frac{\partial \ln \Delta}{\partial x_{j_{1}}} \frac{\partial \ln \Delta}{\partial x_{j_{2}}} .
\end{aligned}
$$

$$
\frac{\partial^{2} \psi^{i}}{\partial x_{j_{1}} \partial x_{j_{2}}}=\Sigma h_{k_{1} k_{2}}^{i} \frac{\partial \psi^{k_{1}}}{\partial x_{j_{1}}} \frac{\partial \phi^{k_{2}}}{\partial x_{j_{2}}}+\frac{1}{N} \frac{\partial \psi^{i}}{\partial x_{j_{1}}} \frac{\partial \ln \Delta}{\partial x_{j_{2}}}+\frac{1}{N} \frac{\partial \psi^{i}}{\partial x_{j_{2}}} \frac{\partial \ln \Delta}{\partial x_{j_{1}}} .
$$

A short calculation then shows that

$$
\begin{aligned}
& \frac{\partial^{3} \psi}{\partial x_{j_{1}} \partial x_{j_{2}} \partial x_{j_{3}}}=\Sigma\left[\mathcal{S} \Sigma h_{k r_{1}}^{i} h_{r_{2} r_{3}}^{k}\right] \frac{\partial \psi^{r_{1}}}{\partial x_{j_{1}}} \frac{\partial \phi^{r_{2}}}{\partial x_{j_{2}}} \frac{\partial \phi^{r_{3}}}{\partial x_{j_{3}}} \\
& +\frac{2}{N} \mathcal{S} \sum \varphi_{k_{1} k_{2}}^{i} \frac{\partial \psi^{k_{1}}}{\partial x_{j_{1}}} \frac{\partial \psi^{k_{2}}}{\partial x_{j_{2}}} \frac{\partial \ln \Delta}{\partial x_{j_{3}}} \\
& +\frac{1}{N^{2}} \mathcal{S} \frac{\partial \phi^{i}}{\partial x_{j_{1}}} \frac{\partial \ln \Delta}{\partial x_{j_{2}}} \frac{\partial \ln \Delta}{\partial x_{j_{3}}}+\mathcal{S} \frac{1}{N} \frac{\partial \phi^{i}}{\partial x_{j_{1}}} \frac{\partial^{2} \ln \Delta}{\partial x_{j_{2}} \partial x_{j_{3}}} \\
& +\frac{\partial \psi^{i}}{\partial x_{j_{1}}}\left[\frac{1}{N^{2}} \frac{\partial \ln \Delta}{\partial x_{j_{2}}} \frac{\partial \ln \Delta}{\partial x_{j_{3}}}-\frac{1}{N} \frac{\partial^{2} \ln \Delta}{\partial x_{j_{2}} \partial x_{j_{3}}}\right] \\
& -\sum h_{k r 1}^{i} h_{r_{2} r_{3}}^{k} \frac{\partial \psi^{r_{1}}}{\partial x_{j_{1}}} \frac{\partial \psi^{r_{2}}}{\partial x_{j_{2}}} \frac{\partial \psi^{r_{3}}}{\partial x_{j_{3}}} \text {. }
\end{aligned}
$$

But this is symmetric, so

$$
\begin{aligned}
& \frac{\partial \phi^{i}}{\partial x_{j_{1}}}\left[\frac{1}{N^{2}} \frac{\partial \ln \Delta}{\partial x_{j_{2}}} \frac{\partial \ln \Delta}{\partial x_{j_{3}}}-\frac{1}{N} \frac{\partial^{2} \ln \Delta}{\partial x_{j_{2}} \partial x_{j_{3}}}\right]\left[\left(\frac{\partial \psi}{\partial x}\right)^{-1}\right]_{s_{1}}^{j_{1}}\left[\left(\frac{\partial \psi}{\partial x}\right)^{-1}\right]_{s_{2}}^{j_{2}}\left[\left(\frac{\partial \psi}{\partial x}\right)^{-1}\right]_{s_{3}}^{j_{3}} \\
& -\left[\sum h_{k r_{1}}^{i} h_{r_{2} r_{3}}^{k} \frac{\partial \psi^{r_{2}}}{\partial x_{j_{2}}} \frac{\partial \psi^{r_{3}}}{\partial x_{j_{3}}}\right] \frac{\partial \psi^{r_{1}}}{\partial x_{j_{1}}}\left[\left(\frac{\partial \psi}{\partial x}\right)^{-1}\right]_{s_{1}}^{j_{1}}\left[\left(\frac{\partial \psi}{\partial x}\right)^{-1}\right]_{s_{2}}^{j_{2}}\left[\left(\frac{\partial \psi}{\partial x}\right)^{-1}\right]_{s_{3}}^{j_{3}} \\
& =\delta_{s_{1}}^{i}\left[\frac{1}{N^{2}} \frac{\partial \ln \Delta}{\partial x_{j_{2}}} \frac{\partial \ln \Delta}{\partial x_{j_{3}}}-\frac{1}{N} \frac{\partial^{2} \ln \Delta}{\partial x_{j_{2}} \partial x_{j_{3}}}\right]\left[\left(\frac{\partial \psi}{\partial x}\right)^{-1}\right]_{s_{2}}^{j_{2}}\left[\left(\frac{\partial \psi}{\partial x}\right)^{-1}\right]_{s_{3}}^{j_{3}} \\
& -\sum h_{k s_{1}}^{i} h_{s_{2} s_{3}}^{k}=\vartheta_{s_{1} s_{2} s_{3}}^{i}
\end{aligned}
$$

is symmetric. But

$$
\Sigma \vartheta^{i}{ }_{i s_{1} s_{2}}=(N-1)\left[\frac{1}{N^{2}} \frac{\partial \ln \Delta}{\partial x_{j_{1}}} \frac{\partial \ln \Delta}{\partial x_{j_{2}}}-\frac{1}{N} \frac{\partial^{2} \ln \Delta}{\partial x_{j_{1}} \partial x_{j_{2}}}\right]\left[\left(\frac{\partial \psi}{\partial x}\right)^{-1}\right]_{s_{1}}^{j_{1}}\left[\left(\frac{\partial \psi}{\partial x}\right)^{-1}\right]_{s_{2}}^{j_{2}}
$$

(since $\Sigma h^{i}{ }_{i k}=0$ )

$$
\begin{aligned}
=\Sigma \vartheta_{s_{1} s_{2}}= & {\left[\frac{1}{N^{2}} \frac{\partial \ln \Delta}{\partial x_{j_{1}}} \frac{\partial \ln \Delta}{\partial x_{j_{2}}}-\frac{1}{N^{2}} \frac{\partial^{2} \ln \Delta}{\partial x_{j_{1}} \partial x_{j_{2}}}\right]\left[\left(\frac{\partial \psi}{\partial x}\right)^{-1}\right]_{s_{1}}^{j_{1}}\left[\left(\frac{\partial \psi}{\partial x}\right)^{-1}\right]_{s_{2}}^{j_{2}} } \\
& -\Sigma h^{i}{ }_{k s_{1}} h^{k}{ }_{i s_{2}}
\end{aligned}
$$


so

$$
(N-2)\left[\frac{1}{N^{2}} \frac{\partial \ln \Delta}{\partial x_{j_{1}}} \frac{\partial \ln \Delta}{\partial x_{j_{2}}}-\frac{1}{N} \frac{=\partial^{2} \ln \Delta}{\partial x_{j_{1}} \partial x_{j_{2}}}\right]=-\Sigma h^{i}{ }_{k s_{1}} h^{k}{ }_{i s_{2}} \frac{\partial \psi^{s_{1}}}{\partial x_{j_{1}}} \frac{\partial \psi^{s_{2}}}{\partial x_{j_{2}}} .
$$

Thus

$$
\begin{aligned}
\frac{\partial^{2} l}{\partial x_{j_{1}} \partial x_{j_{2}}}-\frac{\partial l}{\partial x_{j_{1}}} \frac{\partial l}{\partial x_{j_{2}}}= & \Sigma\left(f_{k} h_{r_{1} r_{2}}^{k}-f_{r_{1}} f_{r_{2}}\right) \frac{\partial \psi^{r_{1}}}{\partial x_{j_{1}}} \frac{\partial \psi^{r_{2}}}{\partial x_{j_{2}}} \\
& +\frac{1}{N-2} \Sigma h^{i}{ }_{k r_{1}} h^{k}{ }_{i r_{2}} \frac{\partial \psi^{r_{1}}}{\partial x_{j_{1}}} \frac{\partial \psi^{r_{2}}}{\partial x_{j_{2}}}-\Sigma e g_{k_{1} k_{2}} \frac{\partial \psi^{k_{1}}}{\partial x_{j_{1}}} \frac{\partial \psi^{k_{2}}}{\partial x_{j_{2}}}
\end{aligned}
$$

by the second boxed-in formula in the discussion of $19 \mathrm{a}$.

Now let $e=0$. Write $\phi^{1}=e^{l}\left(x_{1}+r\left(x_{2}, \cdots, x_{n}\right)\right)$. This solves the first anc: second equations. But $e=0$, so $(N>2) \frac{\partial^{2} l}{\partial x_{j_{1}} \partial x_{j_{2}}}=\frac{\partial l}{\partial x_{j_{1}}} \frac{\partial l}{\partial x_{j_{2}}}$. Looking at the previous calculations, we conclude that it is enough to solve

$$
\frac{\partial^{2} r}{\partial x_{j_{1}} \partial x_{j_{2}}}=e^{-l} \Sigma g_{k_{1} k_{2}} \frac{\partial \psi^{k_{1}}}{\partial x_{j_{1}}} \frac{\partial \psi^{k_{2}}}{\partial x_{j_{2}}} \text {. }
$$

Thus we must show that

$$
\frac{\partial}{\partial x_{j_{3}}}\left[e^{-l} \sum g_{k_{1} k_{2}} \frac{\partial \psi^{k_{1}}}{\partial x_{j_{1}}} \frac{\partial \psi^{k_{2}}}{\partial x_{j_{2}}}\right]=\vartheta_{j_{1} j_{2} j_{3}}
$$

is symmetric. But this is

$$
\begin{aligned}
& e^{-l} \sum g_{k_{1} k_{2}}\left(h^{k_{1}}{ }_{r_{1} r_{2}}+d^{k_{1}}{ }_{r_{1} r_{2}}\right) \frac{\partial \psi^{r_{1}}}{\partial x_{j_{3}}} \frac{\partial \psi^{r_{2}}}{\partial x_{j_{1}}} \frac{\partial \psi^{k_{2}}}{\partial x_{j_{2}}} \\
& \quad+e^{-l} \sum g_{k_{1} k_{2}}\left(h^{k_{1}}{ }_{r_{1} r_{2}}+d^{k_{1}}{ }_{r_{1} r_{2}}\right) \frac{\partial \psi^{r_{1}}}{\partial x_{j_{3}}} \frac{\partial \psi^{r_{2}}}{\partial x_{j_{2}}} \frac{\partial \psi^{k_{2}}}{\partial x_{j_{1}}} \\
& \quad-e^{-l} \sum f_{k} g_{k_{1} k_{2}} \frac{\partial \psi^{k}}{\partial x_{j_{3}}} \frac{\partial \psi^{k_{1}}}{\partial x_{j_{1}}} \frac{\partial \psi^{k_{2}}}{\partial x_{j_{2}}}-e^{-l} \frac{\partial}{\partial x_{j_{3}}}\left(\frac{1}{N} \ln \Delta\right) g_{k_{1} k_{2}} \frac{\partial \psi^{k_{1}}}{\partial x_{j_{1}}} \frac{\partial \psi^{k_{2}}}{\partial x_{j_{2}}} .
\end{aligned}
$$

It suffices if

is symmetric.

$$
\begin{aligned}
& \sum f_{k} g_{k_{1} k_{2}} \frac{\partial \psi^{k}}{\partial x_{j_{3}}} \frac{\partial \psi^{k_{1}}}{\partial x_{j_{1}}} \frac{\partial \psi^{k_{2}}}{\partial x_{j_{2}}}+\sum \frac{\partial}{\partial x_{j_{3}}}\left(\frac{1}{N} \ln \Delta\right) g_{k_{1} k_{2}} \frac{\partial \psi^{k_{1}}}{\partial x_{j_{1}}} \frac{\partial \psi^{k_{2}}}{\partial x_{j_{2}}} \\
& +\sum g_{k_{1} k_{2}}\left(h^{k_{1}}{ }_{r_{1} r_{2}}+d_{r_{1} r_{2}}^{k_{1}}\right) \frac{\partial \psi^{k_{2}}}{\partial x_{j_{3}}} \frac{\partial \psi^{r_{1}}}{\partial x_{j_{1}}} \frac{\partial \psi^{r_{2}}}{\partial x_{j_{2}}}
\end{aligned}
$$

Applying the first boxed-in expression to $f_{k} g_{k_{1} k_{2}}+\sum g_{k r} h_{k_{1} k_{2}}^{r}$, we conclude that it is enough if

$$
\begin{aligned}
\sum \frac{\partial}{\partial x_{j_{3}}} & \left(\frac{1}{N} \ln \Delta\right) g_{k_{1} k_{2}} \frac{\partial \psi^{k_{1}}}{\partial x_{j_{1}}} \frac{\partial \psi^{k_{2}}}{\partial x_{j_{2}}} \\
& +\sum g_{k_{1} k_{2}}\left[\frac{1}{2}\left\{\delta^{k_{1}} d_{r_{1}} d_{r_{2} r_{2}}+\delta^{r_{1}}{ }_{r_{2}} d^{r_{1}}{ }_{r_{1} r_{1}}\right\}\right] \frac{\partial \psi^{k_{1}}}{\partial x_{j_{3}}} \frac{\partial \psi^{r_{1}}}{\partial x_{j_{1}}} \frac{\partial \psi^{r_{2}}}{\partial x_{j_{2}}}
\end{aligned}
$$


is symmetric, and by a now-familiar calculation it is.

Finally we determine the pseudogroup. If $e=f_{i}=g_{i j}=h_{r s}^{i}=0$,

$$
\begin{aligned}
\psi^{i} & =\frac{\sum a_{i j} x_{j}+b_{i}}{\sum c_{j} x_{j}+d} \text { and } l=\frac{1}{N} \ln \Delta, \\
\psi^{1} & =C e^{\frac{1}{N} \ln \Delta}\left(x_{1}+r\left(x_{2}, \cdots, x_{n}\right)\right) \\
& =C(\ln \Delta)^{\frac{1}{N}}\left(x_{1}+r\left(x_{2}, \cdots, x_{n}\right)\right) \\
& =(\text { by lemma } 51) \frac{A x_{1}+r\left(x_{2}, \cdots, x_{n}\right)}{\sum c_{j} x_{j}+d} .
\end{aligned}
$$

Since $\frac{\partial^{2} r}{\partial x_{j_{1}} \partial x_{j_{2}}}=0$, we get $10_{1}$ on the list of pseudogroups.

It remains to look at (18a) on the kernel list. In this case, $N=2$, and $\varphi=\left(\begin{array}{ccc}e & f & g \\ 0 & 0 & 0\end{array}\right), k=\left(\begin{array}{ccc}0 & k & 0 \\ 0 & 0 & 2 k\end{array}\right)$; the equations become

$$
\begin{aligned}
& \frac{\partial^{2} \psi^{1}}{\partial x_{1}{ }^{2}}=e\left(\frac{\partial \psi^{1}}{\partial x_{1}}\right)^{2} \\
& \frac{\partial^{2} \psi^{1}}{\partial x_{1} \partial x_{2}}=e \frac{\partial \psi^{1}}{\partial x_{1}} \frac{\partial \psi^{1}}{\partial x_{2}}+(f+k) \frac{\partial \psi^{1}}{\partial x_{1}} \frac{\partial \psi^{2}}{\partial x_{2}} \\
& \frac{\partial^{2} \psi^{1}}{\partial x_{2}{ }^{2}}=e\left(\frac{\partial \psi^{1}}{\partial x_{2}}\right)^{2}+2(f+k) \frac{\partial \psi^{1}}{\partial x_{2}} \frac{\partial \psi^{2}}{\partial x_{2}}+g\left(\frac{\partial \psi^{2}}{\partial x_{2}}\right)^{2} \\
& \frac{\partial^{2} \psi^{2}}{\partial x_{2}{ }^{2}}=2 k\left(\frac{\partial \psi^{2}}{\partial x_{2}}\right)^{2} .
\end{aligned}
$$

If $e=0$, let $k=-f$. Then $\psi^{1}=x_{1}+l\left(x_{2}\right), \phi^{2}=s\left(x_{2}\right)$. If $f=0$, let $s\left(x_{2}\right)=x_{2}$; $\frac{d^{2} l}{d x_{2}{ }^{2}}=g$. If $f \neq 0, s\left(x_{2}\right)=-\frac{1}{2 k} \ln \left(x_{2}+1\right) ; \frac{d^{2} l}{d x_{2}{ }^{2}}=\frac{g}{4 k^{2}} \frac{1}{\left(x_{2}+1\right)^{2}}$, which can be solved.

If $e \neq 0, \psi^{1}=-\frac{1}{e} \ln \left(x_{1}+1\right)+m\left(x_{2}\right)$; to solve the second equation, we want

$$
\frac{d m}{d x_{2}}=-\frac{f+k}{e} \frac{d \psi^{2}}{d x_{2}}
$$

which can, of course, be solved.

The third equation becomes

$$
\left[-\frac{(f+k)^{2}}{e}+g\right]\left(\frac{d \psi^{2}}{d x_{2}}\right)^{2}=-\frac{\frac{d k}{d x_{2}}}{e} \frac{d \psi^{2}}{d x_{2}}-\frac{f+k}{e} \frac{d^{2} \psi^{2}}{d x_{2}^{2}}
$$

and the last equation

This is equivalent to

$$
\frac{d^{2} \psi^{2}}{d x_{2}^{2}}=2 k\left(\frac{d \phi^{2}}{d x_{2}}\right)^{2}
$$


Let $k\left(x_{2}\right)=x_{2}$. Then

$$
\left(k^{2}-f^{2}+e g\right)\left(\frac{d \psi^{2}}{d x_{2}}\right)^{2}=-\frac{d k}{d x_{2}} \frac{d \psi^{2}}{d x_{2}} .
$$

can be solved, and

$$
\frac{d \psi^{2}}{d x_{2}}=-\frac{1}{x_{2}^{2}-f^{2}+e g}
$$

$$
\frac{d^{2} \psi^{2}}{d x_{2}{ }^{2}}=\frac{2 x_{2}}{\left(x_{2}{ }^{2}-f^{2}+e g\right)^{2}}=2 x_{2}\left(\frac{-1}{x_{2}{ }^{2}-f^{2}+e g}\right)^{2} .
$$

We are done.

Finally, we find the pseudogroup. Then $e=f=g=0$, and

$$
\begin{aligned}
& \phi^{1}=e^{l\left(x_{2}\right)}\left[x_{1}+h\left(x_{2}\right)\right] \\
& \frac{d l}{d x_{2}}=k \frac{d \psi^{2}}{d x_{2}}=\frac{1}{2} \frac{\frac{d^{2} \psi^{2}}{d x_{2}{ }^{2}}}{\frac{d \psi^{2}}{d x_{2}}}=\frac{1}{2} \frac{d}{d x_{2}} \ln \frac{d \psi^{2}}{d x_{2}} .
\end{aligned}
$$

Hence $l=\frac{1}{2} \ln \frac{d \psi^{2}}{d x_{2}}+C$, so

and

$$
\psi^{1}=\left(\frac{d \psi^{2}}{d x_{2}}\right)^{\frac{1}{2}}\left[a x_{1}+h\left(x_{2}\right)\right]
$$

$$
\begin{aligned}
\frac{d}{d x_{2}}[ & \left.\frac{1}{2}\left(\frac{d \psi^{2}}{d x_{2}}\right)^{-\frac{1}{2}} \frac{d^{2} \psi^{2}}{d x_{2}{ }^{2}}\left\{a x_{1}+h\left(x_{2}\right)\right\}+\left(\frac{d \psi^{2}}{d x_{2}}\right)^{\frac{1}{2}} \frac{d h}{d x_{2}}\right] \\
= & \frac{1}{2}\left(-\frac{1}{2}\right)\left(\frac{d \psi^{2}}{d x_{2}}\right)^{-\frac{3}{2}}\left(\frac{d^{2} \psi^{2}}{d x_{2}{ }^{2}}\right)^{2}\left\{a x_{1}+h\left(x_{2}\right)\right\}+\frac{1}{2}\left(\frac{d \psi^{2}}{d x_{2}}\right)^{-\frac{1}{2}} \frac{d^{3} \psi^{2}}{d x_{2}{ }^{3}}\left\{a x_{1}+h\left(x_{2}\right)\right\} \\
& +\frac{1}{2}\left(\frac{d \psi^{2}}{d x_{2}}\right)^{-\frac{1}{2}} \frac{d^{2} \psi^{2}}{d x_{2}{ }^{2}} \frac{d h}{d x_{2}}+\frac{1}{2}\left(\frac{d \psi^{2}}{d x_{2}}\right)^{-\frac{1}{2}} \frac{d^{2} \psi^{2}}{d x_{2}{ }^{2}} \frac{d h}{d x_{2}}+\frac{1}{2}\left(\frac{d \psi^{2}}{d x_{2}}\right)^{\frac{1}{2}} \frac{d^{2} h}{d x_{2}{ }^{2}} \\
= & \frac{\frac{d^{2} \psi}{d x_{2}{ }^{2}}}{\frac{d \psi}{d x_{2}}}\left[\frac{1}{2}\left(\frac{d \psi^{2}}{d x_{2}}\right)^{-\frac{1}{2}} \frac{d^{2} \psi}{d x_{2}{ }^{2}}\left\{a x_{1}+h\left(x_{2}\right)\right\}\left(\frac{d \psi^{2}}{d x_{2}}\right)^{\frac{1}{2}} \frac{d h}{d x_{2}}\right] .
\end{aligned}
$$

Equating the coefficients of $x_{1}$, we have

$$
\frac{d^{3} \psi^{2}}{d x_{2}{ }^{3}} \frac{d \psi^{2}}{d x_{2}{ }^{2}}-\frac{3}{2}\left(\frac{d^{2} \psi^{2}}{d x_{2}{ }^{2}}\right)^{2}=0
$$

This is the Schwarzian differential equation, whose solutions are linear fractional transformations $\frac{a x_{2}+b}{c x_{2}+d}$. The rest of the above equation becomes $\frac{d^{2} h}{d x_{2}{ }^{2}}=0$. Therefore we obtain $10_{1}$ on the list of pseudogroups.

There is an interesting feature in the above calculation. The Schwarzian differential equation, normally a defining equation for $G^{3}$, appears here in the examination of $G^{2}$. 
2. $G^{3}$

Due to the above equivalence result for $G^{2}$, we can assume $g^{2}=\left\langle k^{1}, k^{2}\right\rangle$ and use the lemmas in chapter I to construct $G^{3}$. Thus we are given $\varphi \in \Lambda^{3} k^{2} / k^{2}$ and must solve $\theta_{0}{ }^{3}(\phi)-\varphi \in k^{3}$; the complete pseudogroup consists of those elements $f$ in the pseudogroup defined by $G^{2}$ which satisfy $\theta_{0}{ }^{3}(f) \in k^{3}$.

$\theta^{3}(f)-\varphi \in k^{3}$ can be rewritten

$$
\begin{aligned}
\xi_{j_{1} j_{2} j_{3}}^{i}= & \frac{1}{2}\left[\mathcal{S} \sum \theta^{2}(\xi)_{r_{1} k}^{i} \theta^{2}(\xi)_{r_{2} r_{3}}^{k}\right] \xi^{r_{1}}{ }_{j_{1}} \xi^{r_{2}}{ }_{j_{2}} \xi^{r_{3}}{ }_{j_{3}} \\
& +\frac{1}{2} \sum\left(\varphi_{k_{1} k_{2} k_{3}}^{i}+k_{k_{1} k_{2} k_{3}}^{i}\right) \xi^{k_{1}}{ }_{j_{1}} \xi^{k_{2} j_{2}} \xi^{k_{j_{3}}} .
\end{aligned}
$$

LEMMA 53. Let $\varphi^{i}{ }_{j_{1} j_{2} j_{3}}=\left(\begin{array}{cccc}e & e_{i} & e_{j_{1} j_{2}} & e_{j_{1} j_{2} j_{3}} \\ 0 & 0 & 0 & h^{i} j_{1} j_{2} j_{3}\end{array}\right), \xi^{i}{ }_{k}=\left(\begin{array}{cc}a & b_{i} \\ 0 & d^{i}{ }_{j}\end{array}\right)$. Then

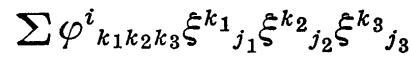

$$
\begin{aligned}
& =\left(\begin{array}{ccc}
e a^{2} e a^{2} b_{j}+\sum a^{2} e_{r} d_{j}^{r}\left[e a b_{j_{1}} b_{j_{2}}+\sum a e_{r} d_{j_{1}}^{r} b_{j_{2}}+\sum a e_{r} d_{j_{2}}^{r_{j_{1}}} b_{j_{1}}+\sum a e_{r_{1} r_{2}} d^{r_{j_{1}}} d^{r_{2}}{ }_{j_{2}}\right] \\
0 & 0 & 0
\end{array}\right. \\
& \left.\begin{array}{c}
{\left[e b_{j_{1}} b_{j_{2}} b_{j_{3}}+\sum e_{r}\left(S b_{j_{1}} b_{j_{2}} d_{j_{3}}^{r}\right)+\sum e_{r_{1} r_{2}}\left(S b_{j_{1}} d_{j_{2}}^{r_{1}} d^{r_{2}{ }_{j_{3}}}\right)+\sum e_{r_{1} r_{2} r_{3}} d_{j_{1}}^{r_{1}} d_{j_{2}}^{r_{2}} d_{j_{3}}^{r_{3}}\right]} \\
\sum h^{i}{ }_{r_{1} r_{2} r_{3}} d^{r_{j_{1}}} d_{j_{j_{2}}} d^{r_{j_{3}}}{ }_{j_{3}}
\end{array}\right) .
\end{aligned}
$$

PROOF. A calculation.

LEMMA 54. Let $\theta^{2}(\phi)^{i}{ }_{j_{1} j_{2}}=\left(\begin{array}{ccc}f & f_{j} & f_{j_{1} j_{2}} \\ 0 & 0 & r^{i} j_{1} j_{2}\end{array}\right)$. Then

$\mathcal{S} \sum \theta^{2}(\psi)^{i}{ }_{j_{1} k} \theta^{2}(\psi)^{k}{ }_{j_{2} j_{3}}=\left(\begin{array}{cccc}3 f^{2} & 3 f f_{j}\left[f f_{j_{1} j_{2}}+\sum f_{r} r_{j_{1} j_{2}}+2 f_{j_{1}} f_{j_{2}}\right] & \mathcal{S}\left(f_{j_{1}} f_{j_{2} j_{3}}+f_{j_{1} r} r_{j_{2} j_{3}}^{r}\right. \\ 0 & 0 & 0 & \mathcal{S} \sum r_{j_{1} k} r_{j_{2} j_{3}}\end{array}\right)$.

ProOF. A calculation.

LEMMA 55. The equation $\theta^{3}(\psi)-\varphi \in k^{3}$ is equivalent to the following set of equations, if

$$
\varphi+k^{3}=\left(\begin{array}{cccc}
e & e_{i} & e_{i j} & e_{i j k} \\
0 & 0 & 0 & h^{i}{ }_{j k s}
\end{array}\right), \quad \theta^{2}(f)=\left(\begin{array}{ccc}
f & f_{i} & f_{i j} \\
0 & 0 & r^{i}{ }_{j k}
\end{array}\right)
$$

(A) $2 \frac{\partial^{3} \psi^{1}}{\partial x_{1}{ }^{3}}=\left(3 f^{2}+e\right)\left(\frac{\partial \psi^{1}}{\partial x_{1}}\right)^{3}$

(B) $2 \frac{\partial^{3} \psi^{1}}{\partial x_{1}{ }^{2} \partial x_{j}}=\left(3 f^{2}+e\right)\left(\frac{\partial \psi^{1}}{\partial x_{1}}\right)^{2}\left(\frac{\partial \psi^{1}}{\partial x_{j}}\right)+\sum_{r}\left(3 f f_{r}+e_{r}\right)\left(\frac{\partial \psi^{1}}{\partial x_{1}}\right)^{2}\left(\frac{\partial \psi^{r}}{\partial x_{j}}\right)$

(C) $2 \frac{\partial^{3} \psi^{1}}{\partial x_{1} \partial x_{j_{1}} \partial x_{j_{2}}}$

$$
=\left(3 f^{2}+e\right)\left(\frac{\partial \psi^{1}}{\partial x_{1}}\right)\left(\frac{\partial \psi^{1}}{\partial x_{j_{1}}}\right)\left(\frac{\partial \psi^{1}}{\partial x_{j_{2}}}\right)
$$




$$
\begin{aligned}
& +\Sigma\left(3 f f_{k}+e_{k}\right)\left[\left(\frac{\partial \psi^{1}}{\partial x_{1}}\right)\left(\frac{\partial \psi^{1}}{\partial x_{j_{1}}}\right)\left(\frac{\partial \psi^{k}}{\partial x_{j_{2}}}\right)+\left(\frac{\partial \psi^{1}}{\partial x_{1}}\right)\left(\frac{\partial \psi^{1}}{\partial x_{j_{2}}}\right)\left(\frac{\partial \psi^{k}}{\partial x_{j_{1}}}\right)\right] \\
& +\Sigma\left(f f_{k_{1} k_{2}}+\Sigma f_{8} r_{k_{1} k_{2}}^{s}+2 f_{k_{1}} f_{k_{2}}+e_{k_{1} k_{2}}\right)\left(\frac{\partial \psi^{1}}{\partial x_{1}}\right)\left(\frac{\partial \psi^{k_{1}}}{\partial x_{j_{1}}}\right)\left(\frac{\partial \psi^{k_{2}}}{\partial x_{j_{2}}}\right)
\end{aligned}
$$

(D) $2 \frac{\partial^{3} \psi^{1}}{\partial x_{j_{1}} \partial x_{j_{2}} \partial x_{j_{3}}}$

$$
\begin{aligned}
= & \left(3 f^{2}+e\right)\left(\frac{\partial \psi^{1}}{\partial x_{j_{1}}}\right)\left(\frac{\partial \psi^{1}}{\partial x_{j_{2}}}\right)\left(\frac{\partial \psi^{1}}{\partial x_{j_{3}}}\right)+\Sigma\left(3 f f_{k}+e_{k}\right) \mathcal{S}\left[\left(\frac{\partial \psi^{1}}{\partial x_{j_{1}}}\right)\left(\frac{\partial \psi^{1}}{\partial x_{j_{2}}}\right)\left(\frac{\partial \psi^{k}}{\partial x_{j_{3}}}\right)\right] \\
& +\Sigma\left(f f_{k_{1} k_{2}}+\Sigma f_{s} r_{k_{1} k_{2}}+2 f_{k_{1}} f_{k_{2}}+e_{k_{1} k_{2}}\right) \mathcal{S}\left[\left(\frac{\partial \psi^{1}}{\partial x_{j_{1}}}\right)\left(\frac{\partial \psi^{k_{1}}}{\partial x_{j_{2}}}\right)\left(\frac{\partial \psi^{k_{2}}}{\partial x_{j_{3}}}\right)\right] \\
& +\Sigma\left[\mathcal{S}\left\{f_{k_{1}} f_{k_{2} k_{3}}+\Sigma f_{k_{1} s} r_{k_{2} k_{3}}^{s}+e_{k_{1} k_{2} k_{3}}\right]\left(\frac{\partial \psi^{k_{1}}}{\partial x_{j_{1}}}\right)\left(\frac{\partial \psi^{k_{2}}}{\partial x_{j_{2}}}\right)\left(\frac{\partial \psi^{k_{3}}}{\partial x_{j_{3}}}\right)\right.
\end{aligned}
$$

(E) $2 \frac{\partial^{3} \psi^{i}}{\partial x_{j_{1}} \partial x_{j_{2}} \partial x_{j_{3}}}=\left[\mathcal{S} \Sigma r_{k_{1 s}}^{i} r_{k_{2} k_{3}}+h_{k_{1} k_{2} k_{3}}^{i}\right]\left(\frac{\partial \psi^{k_{1}}}{\partial x_{j_{1}}}\right)\left(\frac{\partial \psi^{k_{2}}}{\partial x_{j_{2}}}\right)\left(\frac{\partial \psi^{k_{3}}}{\partial x_{j_{3}}}\right)$

Proof. A calculation based on lemmas 53 and 54 .

LEMMA 56. If $e$ is arbitrary in $k^{3}$, (A) is irrelevant; if $e_{i}$ is arbitrary, (B), is irrelevant; if $e_{i j}$ is arbitrary, (C) is irrelevant; if $e_{i j k}$ is arbitrary, (D) is. irrelevant; if $h^{i}{ }_{j k s}$ is arbitrary, (E) is irrelevant.

Proof. The proof is exactly the same as the proof of lemma 49.

LEMMA 57.

$$
\begin{aligned}
& \theta^{2}(\psi)=\left(\begin{array}{lll}
f & f_{i} & f_{i j} \\
0 & 0 & r_{j k}{ }_{j k}
\end{array}\right) \\
&=\left(\begin{array}{l}
\frac{\partial^{2} \psi^{1}}{\partial x_{1}{ }^{2}}\left\{\left[\left(\frac{\partial \psi}{\partial x}\right)^{-1}\right]_{1}^{1}\right\}^{2} \\
0
\end{array}\right. \\
& \frac{\partial^{2} \psi^{1}}{\partial x_{1}{ }^{2}}\left[\left(\frac{\partial \psi}{\partial x}\right)^{-1}\right]_{1}^{1}\left[\left(\frac{\partial \psi}{\partial x}\right)^{-1}\right]_{j}^{1}+\Sigma \frac{\partial^{2} \psi^{1}}{\partial x_{1} \partial x_{k}}\left[\left(\frac{\partial \psi}{\partial x}\right)^{-1}\right]_{j}^{k}\left[\left(\frac{\partial \psi}{\partial x}\right)^{-1}\right]_{1}^{1} \\
& \frac{\partial^{2} \psi^{1}}{\partial x_{1}{ }^{2}}\left[\left(\frac{\partial \psi}{\partial x}\right)^{-1}\right]_{j_{1}}^{1}\left[\left(\frac{\partial \psi}{\partial x}\right)^{-1}\right]_{j_{2}}^{1}+\Sigma \frac{\partial^{2} \psi^{1}}{\partial x_{1} \partial x_{k}}\left\{\left[\left(\frac{\partial \psi}{\partial x}\right)^{-1}\right]_{j_{1}}^{k}\left[\left(\frac{\partial \psi}{\partial x}\right)^{-1}\right]_{j_{2}}^{1}\right. \\
& {\left.\left[\left(\frac{\partial \psi}{\partial x}\right)^{-1}\right]_{j_{2}}^{k}\left[\left(\frac{\partial \psi}{\partial x}\right)^{-1}\right]_{j_{1}}^{1}\right\}+\Sigma \frac{\partial^{2} \psi^{i}}{\partial x_{k_{1}} \partial x_{k_{2}}}\left[\left(\frac{\partial \psi}{\partial x}\right)^{-1}\right]_{j_{1}}^{k_{1}}\left[\left(\frac{\partial \psi}{\partial x}\right)^{-1}\right]_{j_{2}}^{k_{2}} } \\
& \frac{\partial^{2} \psi^{i}}{\partial x_{k_{1}} \partial x_{k_{2}}}\left[\left(\frac{\partial \psi}{\partial x}\right)^{-1}\right]_{j_{1}}^{k_{1}}\left[\left(\frac{\partial \psi}{\partial x}\right)^{-1}\right]_{j_{2}}^{k_{2}}
\end{aligned}
$$

ProOF. A consequence of lemma 47. 
Now we begin a step by step calculation; we examine those kernel sequences on our list not of the form $k^{2}, \Lambda^{3} k^{2}, \cdots$.

The first occurs in (2). Here $\varphi=\left(\begin{array}{llll}0 & 0 & 0 & \varphi_{j_{1} j_{2} j_{3}} \\ 0 & 0 & 0 & 0\end{array}\right), k^{3}=0$. Moreover, the $G^{2}$ pseudogroup is

$$
\begin{aligned}
& \psi^{1}=c x_{1}+g\left(x_{2}, \cdots, x_{n}\right) \\
& \psi^{i}=\Sigma a_{i j} x_{j}+b_{i} .
\end{aligned}
$$

Hence $\frac{\partial^{2} \psi^{1}}{\partial x_{1}{ }^{2}}=\frac{\partial^{2} \psi^{1}}{\partial x_{1} \partial x_{j}}=\frac{\partial^{2} \psi^{i}}{\partial x_{j_{1}} \partial x_{j_{2}}}=0$, so

$$
\left(\begin{array}{ccc}
f & f_{i} & f_{i j} \\
0 & 0 & r_{j k}^{i}
\end{array}\right)=\left(\begin{array}{ccc}
0 & 0 & \sum \frac{\partial^{2} g}{\partial x_{k_{1}} \partial x_{k_{2}}}\left(a^{-1}\right)^{k_{1}}{ }_{j_{1}}\left(a^{-1}\right)^{k_{2}} j_{j_{2}} \\
0 & 0 & 0
\end{array}\right) .
$$

Equations (A), (B), (C), and (E) are trivial, and (D) becomes

But Ewe can pick

$$
2 \frac{\partial^{3} g}{\partial x_{j_{1}} \partial x_{j_{2}} \partial x_{j_{3}}}=\sum \varphi_{k_{1} k_{2} k_{3}} a_{j_{1}}^{k_{1}} a_{j_{2}}^{k_{2}} a_{j_{3}}^{k_{3}}
$$

$$
\begin{aligned}
& \psi^{1}=x_{1}+g\left(x_{2}, \cdots, x_{n}\right) \\
& \psi^{i}=x_{i}
\end{aligned}
$$

and it suffices if $\frac{\partial^{3} g}{\partial x_{j_{1}} \partial x_{j_{2}} \partial x_{j_{3}}}=\varphi_{j_{1} j_{2} j_{3}}$ which is easily done. The pscudogroup is clearly

$$
\left\{\begin{array}{l}
y_{1}=c x_{1}+P_{2}\left(x_{2}, \cdots, x_{n}\right) \\
y_{i}=\Sigma a_{i j} x_{j}+b_{i}
\end{array}\right.
$$

Next, examine (3); here $k^{3}=\left(\begin{array}{llll}0 & 0 & 0 & a \\ 0 & 0 & 0 & 0\end{array}\right)$ and $\varphi=\left(\begin{array}{cccc}0 & 0 & 0 & 0 \\ 0 & 0 & 0 & s\end{array}\right)$. The $G^{2}$ pseudogroup is

Then

$$
\left\{\begin{array}{l}
y_{1}=c x_{1}+g\left(x_{2}\right) \\
y_{i}=f\left(x_{2}\right)
\end{array}\right.
$$

$$
\left(\begin{array}{ccc}
f & f_{i} & f_{i j} \\
0 & 0 & r_{j k}^{i}
\end{array}\right)=\left(\begin{array}{lll}
0 & 0 & \frac{\partial^{2} g}{\partial x_{2}{ }^{2}}\left(\frac{\partial f}{\partial x_{2}}\right)^{-2} \\
0 & 0 & \frac{\partial^{2} f}{\partial x_{2}{ }^{2}}\left(\frac{\partial f}{\partial x_{2}}\right)^{-2}
\end{array}\right)
$$

equations (A), (B), and (C) are trivial, and (D) is trivial by lemma 56 , so we must solve

$$
\begin{aligned}
2 \frac{\partial^{3} f}{\partial x_{2}{ }^{3}} & =\left\{\left[3 \frac{\partial^{2} f}{\partial x_{2}{ }^{2}}\left(\frac{\partial f}{\partial x_{2}}\right)^{-2}\right]^{2}+s\right\}\left(\frac{\partial f}{\partial x_{2}}\right)^{3} \\
& =3\left(\frac{\partial^{2} f}{\partial x_{2}{ }^{2}}\right)^{2}\left(\frac{\partial f}{\partial x_{2}}\right)^{-1}+s\left(\frac{\partial f}{\partial x_{2}}\right)^{3}
\end{aligned}
$$


or

$$
\frac{d^{3} f}{d x_{2}^{3}} \frac{d f}{d x_{2}}-\frac{3}{2}\left(\frac{d^{2} f}{d x_{2}^{2}}\right)^{2}=\frac{s}{2}\left(\frac{d f}{d x_{2}}\right)^{4} .
$$

But this can be solved and if $s=0$, it is the Schwarzian differential equation; the pseudogroup is

$$
\left\{\begin{array}{l}
y_{1}=A x_{1}+g\left(x_{2}\right) \\
y_{2}=\frac{a x_{2}+b}{c x_{2}+d}
\end{array}\right.
$$

which is (3) on the list of pseudogroups.

The next kernel sequence is (7). Here $k^{3}=\left(\begin{array}{llll}0 & 0 & 0 & e_{j k r} \\ 0 & 0 & 0 & 0\end{array}\right), \varphi=\left(\begin{array}{llll}0 & 0 & \varphi_{i j} & 0 \\ 0 & 0 & 0 & 0\end{array}\right)$, and the $G^{2}$ pseudogroup is

Then

$$
\left\{\begin{array}{l}
y_{1}=x_{1} g\left(x_{2}, \cdots, x_{n}\right)+k\left(x_{2}, \cdots, x_{n}\right) \\
y_{i}=\sum a_{i j} x_{j}+b_{i} .
\end{array}\right.
$$

$$
\left(\begin{array}{ccc}
f & f_{i} & f_{i j} \\
0 & 0 & r_{j k}^{i}
\end{array}\right)=\left(\begin{array}{ccc}
0 & \frac{\partial g}{\partial x_{k}}\left(a^{-1}\right)^{k}{ }_{j} g^{-1} & * \\
0 & 0 & 0
\end{array}\right)
$$

(* is immaterial).

Equations (A), (B), and (E) are trivial, and (D) is immaterial by lemma 56 , so we only have

$$
\begin{aligned}
2 \frac{\partial^{2} g}{\partial x_{j_{1}} \partial x_{j_{2}}} & =2\left[\sum \frac{\frac{\partial g}{\partial x_{k_{1}}}}{g} \frac{\frac{\partial g}{\partial x_{k_{2}}}}{g}-\left(a^{-1}\right)^{k_{1} r_{1}}\left(a^{-1}\right)^{k_{2}}{ }_{r_{2}} a_{r_{1} j_{1}} a_{r_{2} j_{2}} g+\sum \varphi_{r_{1} r_{2}} a_{r_{1} j_{1}} a_{r_{2} j_{2}} g\right] \\
& =2\left[g \frac{\frac{\partial g}{\partial x_{j_{1}}}}{g} \frac{\frac{\partial g}{\partial x_{j_{2}}}}{g}+g \sum \varphi_{r_{1} r_{2}} a_{r_{1} j_{1}} a_{r_{2} j_{2}}\right] .
\end{aligned}
$$

Letting $g=e^{h}$, this reads

or

$$
2\left[e^{h} \frac{\partial^{2} h}{\partial x_{j_{1}} \partial x_{j_{2}}}+e^{h} \frac{\partial h}{\partial x_{j_{1}}} \frac{\partial h}{\partial x_{j_{2}}}\right]=2\left[e^{h} \frac{\partial h}{\partial x_{j_{1}}} \frac{\partial h}{\partial x_{j_{2}}}+e^{h} \sum \varphi_{r_{1} r_{2}} a_{r_{1} j_{1}} a_{r_{2} j_{2}}\right]
$$

$$
\frac{\partial^{2} h}{\partial x_{j_{1}} \partial x_{j_{2}}}=\frac{1}{2} \Sigma \varphi_{r_{1} r_{2}} a_{r_{1} j_{1}} a_{r_{2} j_{2}}
$$

so to solve the equivalence problem we let $\left\{\begin{array}{l}\psi^{1}=x_{1} e^{h} \\ \psi^{i}=x_{i}\end{array}\right.$ with $\frac{\partial^{2} h}{\partial x_{j_{1}} \partial x_{j_{2}}}=\frac{1}{2} \varphi_{j_{1} j_{2}-}$

Similarly the pseudogroup is

$$
\left\{\begin{array}{l}
y_{1}=e^{P_{1}\left(x_{2}, \cdots, x_{n}\right)}+k\left(x_{2}, \cdots, x_{n}\right) \\
y_{i}=\Sigma a_{i j} x_{j}+b_{i}
\end{array}\right.
$$


which is $6_{1}$ on the list of pseudogroups.

Next consider (8) on the list of kernels; $k^{2}=\left(\begin{array}{llll}0 & 0 & a & b \\ 0 & 0 & 0 & 0\end{array}\right), \varphi=\left(\begin{array}{llll}0 & 0 & 0 & 0 \\ 0 & 0 & 0 & s\end{array}\right)$; the $G^{2}$ pseudogroup is

But

$$
\left\{\begin{array}{l}
y_{1}=x_{1} g\left(x_{2}\right)+h\left(x_{2}\right) \\
y_{2}=f\left(x_{2}\right)
\end{array}\right.
$$

$$
\left(\begin{array}{ccc}
f & f_{i} & f_{i j} \\
0 & 0 & r_{j k}^{i}
\end{array}\right)=\left(\begin{array}{ccc}
0 & * & * \\
0 & 0 & \frac{d^{2} f}{d x_{2}^{2}}\left(\frac{d f}{d x_{2}}\right)^{-2}
\end{array}\right)
$$

so (using lemma 56), (A), (B), (C), and (D) are trivial, and

$$
2 \frac{d^{3} f}{d x_{2}{ }^{3}}=3\left[\frac{d^{2} f}{d x_{2}{ }^{2}}\left(\frac{d f}{d x_{2}}\right)^{-2}\right]^{2}\left(\frac{d f}{d x_{2}}\right)^{3}+s\left(\frac{d f}{d x_{2}}\right)^{3},
$$

an equation that has already occurred. The pseudogroup is (7) on the list of pseudogroups.

In $(12), k^{3}=\left(\begin{array}{cccc}0 & e_{j} & e_{j k} & e_{j k r} \\ 0 & 0 & 0 & 0\end{array}\right), \varphi=\left(\begin{array}{llll}s & 0 & 0 & 0 \\ 0 & 0 & 0 & 0\end{array}\right)$ and the $G^{2}$ pseudogroup is $\left\{\begin{array}{l}\psi^{1}=f\left(x_{1}, \cdots, x_{n}\right) \\ \psi^{i}=\Sigma a_{i j} x_{j}+b_{i}\end{array}\right.$. Then

and

$$
\left(\begin{array}{ccc}
f & f_{i} & f_{i j} \\
0 & 0 & r_{j k}^{i}
\end{array}\right)=\left(\begin{array}{ccc}
\frac{\partial^{2} f}{\partial x_{1}{ }^{2}}\left\{\left[\left(\frac{\partial \psi}{\partial x}\right)^{-1} \cdot\right]_{1}^{1}\right\}^{2} & * & * \\
0 & 0 & 0
\end{array}\right)
$$

$$
2 \frac{\partial^{3} f}{\partial x_{1}{ }^{3}}=\left\{3\left[\frac{\partial^{2} f}{\partial x_{1}{ }^{2}}\left\{\left[\left(\frac{\partial \psi}{\partial x}\right)^{-1}\right]_{1}^{1}\right\}^{2}\right]^{2}+s\right\}\left(\frac{\partial f}{\partial x_{1}}\right)^{3} .
$$

If $a_{i j}=\delta^{i}{ }_{j}, b_{i}=0, f\left(x_{1}, \cdots, x_{n}\right)=f\left(x_{1}\right)$, we have our old familiar equation, which can be solved. To find the pseudogroup, let $s=0$ :

$$
\frac{\partial^{3} f}{\partial x_{1}^{3}}-\frac{3}{2}\left(\frac{\partial^{2} f}{\partial x_{1}}\right)^{2}\left\{\left[\left(\frac{\partial \psi}{\partial x}\right)^{-1}\right]_{1}^{1}\right\}^{4}\left(\frac{\partial f}{\partial x_{1}}\right)^{3}=0 .
$$

But clearly $\left[\left(\frac{\partial \psi}{\partial x}\right)^{-1}\right]_{1}^{1}=\left(\frac{\partial f}{\partial x_{1}}\right)^{-1}$, so this says that for each $x_{2}, \cdots, x_{n}, f$ is linear fractional; hence we have (12) on the list of pseudogroups.

In (13), (14), (15), and (16), we clearly have the same situation, since the equation on $\frac{\partial^{3} \psi^{i}}{\partial x_{j_{1}} \partial x_{j_{2}} \partial x_{j_{3}}}$ is a consequence of the $G^{2}$ equations.

Next consider (18). $\quad k^{3}=\left(\begin{array}{cccc}0 & 0 & 0 & e \\ 0 & 0 & 0 & 0\end{array}\right), \varphi=\left(\begin{array}{cccc}0 & 0 & \lambda s & 0 \\ 0 & 0 & 0 & s\end{array}\right)$, and the pseudogroup given by $G^{2}$ is

$$
\left\{\begin{array}{l}
y_{1}=C x_{1}\left(-\frac{\partial f}{\partial x_{2}}\right)^{2}+h\left(x_{2}\right) \\
y_{2}=f\left(x_{2}\right)
\end{array}\right.
$$


Then

$$
\left(\begin{array}{ccc}
f & f_{i} & f_{i j} \\
0 & 0 & r^{i}{ }_{j k}
\end{array}\right)=\left(\begin{array}{ccc}
0 \frac{d}{d x_{2}}\left[C\left(\frac{d f}{d x_{2}}\right)^{\lambda}\right]\left(\frac{d f}{d x_{2}}\right)^{-1} \frac{1}{C\left(\frac{d f}{d x_{2}}\right)^{2}} & * \\
0 & 0 & \frac{d^{2} f}{d x_{2}{ }^{2}}\left(\frac{d f}{d x_{2}}\right)^{-2}
\end{array}\right)
$$

so equations (A), (B), and (D) are trivial. We have

(C) $2 \frac{d^{2}}{d x_{2}^{2}}\left[C\left(\frac{d f}{d x_{2}}\right)^{\lambda}\right]=\left[\frac{\lambda}{2}\left(\frac{d f}{d x_{2}}\right)^{-4}\left(\frac{d^{2} f}{d x_{2}^{2}}\right)^{2}\left(\frac{d f}{d x_{2}}\right)^{2}\right.$

$$
\left.+2 \lambda^{2}\left(\frac{d f}{d x_{2}}\right)^{-4}\left(\frac{d^{2} f}{d x_{2}^{2}}\right)^{2}\left(\frac{d f}{d x_{2}}\right)^{2}+s \lambda\left(\frac{d f}{d x_{2}}\right)^{2}\right] C\left(\frac{d f}{d x_{2}}\right)^{2}
$$

(E). $2 \frac{d^{3} f}{d x_{2}^{3}}=3\left[\frac{d^{2} f}{d x_{2}{ }^{2}}\left(\frac{d f}{d x_{2}}\right)^{-2}\right]^{2}\left(\frac{d f}{d x_{2}}\right)^{-8}+s\left(\frac{d f}{d x_{2}}\right)^{-3}$.

This last equation is already famous. The first equation may be rewritten

$$
\begin{aligned}
& 2 \lambda(\lambda-1)\left(\frac{d f}{d x_{2}}\right)^{-2}\left(\frac{d^{2} f}{d x_{2}{ }^{2}}\right)^{2}+2 \lambda\left(\frac{d f}{d x_{2}}\right)^{-1} \frac{d^{3} f}{d x_{2}{ }^{3}} \\
& =\lambda\left(\frac{d^{2} f}{d x_{2}{ }^{2}}\right)^{2}\left(\frac{d f}{d x_{2}}\right)^{-2}+2 \lambda^{2}\left(\frac{d^{2} f}{d x_{2}{ }^{2}}\right)^{2}\left(\frac{d f}{d x_{2}}\right)^{-2}+\lambda s\left(\frac{d f}{d x_{2}}\right)^{2}
\end{aligned}
$$

or

$$
\lambda\left[\frac{d^{3} f}{d x_{2}{ }^{3}} \frac{d f}{d x_{2}}-\frac{3}{2}\left(\frac{d^{2} f}{d x_{2}{ }^{2}}\right)^{2}-\frac{s}{2}\left(\frac{d f}{d x_{2}}\right)^{4}\right]=0
$$

and so is implied by (E). The pseudogroup is clearly 10 on the pseudogroup list.

Consider (18b); then $\lambda=1, k^{2}=0, \varphi=\left(\begin{array}{llll}0 & 0 & s & e \\ 0 & 0 & 0 & s\end{array}\right)$ and the $G^{2}$ pseudogroup is as above. We may, in fact, use the results of the above calculation; there is one additional equation, (D), to consider. But

$$
\begin{aligned}
& \left(\begin{array}{ccc}
f & f_{i} & f_{i j} \\
0 & 0 & r_{j k}^{i}
\end{array}\right)=\left(\begin{array}{ccc}
0 & \frac{d^{2} f}{d x_{2}^{2}}\left(\frac{d f}{d x_{2}}\right)^{-2} & * \\
0 & 0 & \frac{d^{2} f}{d x_{2}^{2}}\left(\frac{d f}{d x_{2}}\right)^{-2}
\end{array}\right) \\
& *=2 C \frac{d^{2} f}{d x_{2}^{2}}\left(\frac{d f}{d x_{2}}\right)^{-1}\left[-x_{1} \frac{d^{2} f}{d x_{2}^{2}}\left(\frac{d f}{d x_{2}}\right)^{-2}-\frac{d h}{d x_{2}}\left(\frac{d f}{d x_{2}}\right)^{-2} \frac{1}{C}\right] \\
& +\left[C x_{1} \frac{d^{3} f}{d x_{2}^{3}}+\frac{d^{2} h}{d x_{2}^{2}}\right]\left(\frac{d f}{d x_{2}}\right)^{-2} \\
& =-2 C x_{1}\left(\frac{d^{2} f}{d x_{2}^{2}}\right)^{2}\left(\frac{d f}{d x_{2}}\right)^{-3}-2 \frac{d^{2} f}{d x_{2}^{2}}\left(\frac{d f}{d x_{2}}\right)^{-3} \frac{d h}{d x_{2}} \\
& +C x_{1} \frac{d^{3} f}{d x_{2}^{3}}\left(\frac{d f}{d x_{2}}\right)^{-2}+\frac{d^{2} h}{d x_{2}^{2}}\left(\frac{d f}{d x_{2}}\right)^{-2}
\end{aligned}
$$


and (D) becomes

$$
\begin{aligned}
& 2\left[C x_{1} \frac{d^{4} f}{d x_{2}{ }^{4}}+\frac{d^{3} f}{d x_{2}{ }^{3}}\right] \\
& =3\left[3\left(\frac{d^{2} f}{d x_{2}{ }^{2}}\right)^{2}\left(\frac{d f}{d x_{2}}\right)^{-4}+s\right]\left(\frac{d f}{d x_{2}}\right)^{2}\left[C x_{1} \frac{d^{2} f}{d x_{2}{ }^{2}}+\frac{d h}{d x_{2}}\right] \\
& +6 \frac{d^{2} f}{d x_{2}{ }^{2}}\left(\frac{d f}{d x_{2}}\right)\left[-2 C x_{1}\left(\frac{d^{2} f}{d x_{2}{ }^{2}}\right)^{2}\left(\frac{d f}{d x_{2}}\right)^{-3}-2 \frac{d^{2} f}{d x_{2}{ }^{2}}\left(\frac{d f}{d x_{2}}\right)^{-3} \frac{d h}{d x_{2}}\right. \\
& \left.+C x_{1} \frac{d^{3} f}{d x_{2}{ }^{3}}\left(\frac{d f}{d x_{2}}\right)^{-2}+\frac{d^{2} h}{d x_{2}{ }^{2}}\left(\frac{d f}{d x_{2}}\right)^{-2}\right]+e\left(\frac{d f}{d x_{2}}\right)^{3}
\end{aligned}
$$

or

$$
\begin{gathered}
C x_{1}\left[2 \frac{d^{4} f}{d x_{2}{ }^{4}}+3\left(\frac{d^{2} f}{d x_{2}{ }^{2}}\right)^{3}\left(\frac{d f}{d x_{2}}\right)^{-2}-3 s \frac{d^{2} f}{d x_{2}{ }^{2}}\left(\frac{d f}{d x_{2}}\right)^{2}-6 \frac{d^{2} f}{d x_{2}{ }^{2}} \frac{d^{3} f}{d x_{2}{ }^{3}}\left(\frac{d f}{d x_{2}}\right)^{-1}\right] \\
+2 \frac{d^{3} h}{d x_{2}{ }^{3}}-9\left(\frac{d^{2} f}{d x_{2}{ }^{2}}\right)^{2}\left(\frac{d f}{d x_{2}}\right)^{-2} \frac{d h}{d x_{2}}-3 s \frac{d h}{d x_{2}}\left(\frac{d f}{d x_{2}}\right)^{2}-e\left(\frac{d f}{d x_{2}}\right)^{3} \\
+12\left(\frac{d^{2} f}{d x_{2}{ }^{2}}\right)^{2}\left(\frac{d f}{d x_{2}}\right)^{-2} \frac{d h}{d x_{2}}-6 \frac{d^{2} f}{d x_{2}{ }^{2}} \frac{d^{2} h}{d x_{2}{ }^{2}}\left(\frac{d f}{d x_{2}}\right)^{-1}=0 .
\end{gathered}
$$

But

$$
\frac{d^{3} f}{d x_{2}{ }^{3}} \frac{d f}{d x_{2}}-\frac{3}{2}\left(\frac{d^{2} f}{d x_{2}{ }^{2}}\right)^{2}=\frac{s}{2}\left(\frac{d f}{d x_{2}}\right)^{4}
$$

so the coefficient of $C x_{1}$ is easily shown to vanish. The equivalence problem then can be solved by known facts about linear differential equations. To find the pseudogroup, we let $s=e=0$; the above equation becomes

But

$$
2 \frac{d^{3} h}{d x_{2}{ }^{3}}-c \frac{d^{2} f}{d x_{2}{ }^{2}}\left(\frac{d f}{d x_{2}}\right)^{-1} \frac{d^{2} h}{d x_{2}{ }^{2}}+3\left(\frac{d^{2} f}{d x_{2}{ }^{2}}\right)^{2}\left(\frac{d f}{d x_{2}}\right)^{-2} \frac{d h}{d x_{2}}=0 \text {. }
$$

$$
\left\{\begin{array}{l}
y_{1}=\frac{A x_{1}}{\left(c x_{2}+d\right)^{2}}+h\left(x_{2}\right) \\
y_{2}=\frac{a x_{2}+b}{c x_{2}+d}
\end{array}\right.
$$

and

$$
\frac{\frac{d^{2} f}{d x_{2}{ }^{2}}}{\frac{d f}{d x_{2}}}=\frac{-2 c}{c x_{2}+d},
$$

so

$$
\frac{d^{3} h}{d x_{2}{ }^{3}}+\frac{6 c}{c x_{2}+d} \frac{d^{2} h}{d x_{2}^{2}}+\frac{6 c^{2}}{\left(c x_{2}+d\right)^{2}} \frac{d h}{d x_{2}}=0 \text {. }
$$

Writing $h\left(x_{2}\right)=\frac{\tilde{h}\left(x_{2}\right)}{\left(c x_{2}+d\right)^{2}}$, this equation reads $\frac{d^{3} \tilde{h}}{d x_{2}^{3}}=0$, so 


$$
\left\{\begin{array}{l}
y_{1}=\frac{A x_{1}+P_{2}\left(x_{2}\right)}{\left(c x_{2}+d\right)^{2}} \\
y_{2}=\frac{a x_{2}+b}{c x_{2}+d}
\end{array}\right.
$$

which is $10_{2}$ on the list of pseudogroups.

Finally consider (19b). Then $k^{3}=0, \varphi=\left(\begin{array}{llll}0 & 0 & 0 & \varphi_{j k r} \\ 0 & 0 & 0 & 0\end{array}\right)$, and the $G^{2}$ pseudogroup is

$$
\left\{\begin{array}{l}
y_{1}=\frac{A x_{1}+h\left(x_{2}, \cdots, x_{n}\right)}{\sum c_{j} x_{j}+d} \\
y_{i}=\frac{\sum a_{i j} x_{j}+b_{i}}{\sum c_{j} x_{j}+d} .
\end{array}\right.
$$

But if $\varphi=0$, and $k^{3}=\left(\begin{array}{llll}0 & 0 & 0 & e_{i j k} \\ 0 & 0 & 0 & 0\end{array}\right)$, this is the trivial extension of $G^{2}$, so all equations hold. The only difference occurs in equation (D); there the situation is almnst exactly that of (18b), so we only sketch it.

$$
2 \frac{\partial^{3} \psi^{1}}{\partial x_{j_{1}} \partial x_{j_{2}} \partial x_{j_{3}}}=2 x_{1} \frac{\partial^{3}}{\partial x_{j_{1}} \partial x_{j_{2}} \partial x_{j_{3}}}\left(\frac{A}{\sum c_{j} x_{j}+d}\right)+2 \frac{\partial^{3}}{\partial x_{j_{1}} \partial x_{j_{2}} \partial x_{j_{3}}}\left(\frac{h}{\sum c_{j} x_{j}+d}\right) ;
$$

a simple calculation shows the right hand side is

$$
\begin{aligned}
2 x_{1} \frac{\partial^{3}}{\partial x_{j_{1}} \partial x_{j_{2}} \partial x_{j_{3}}}\left(\frac{A}{\sum c_{j} x_{j}+d}\right) & +2 \frac{\partial^{3}}{\partial x_{j_{1}} \partial x_{j_{2}} \partial x_{j_{3}}}\left(\frac{h}{\sum c_{j} x_{j}+d}\right) \\
& -2 \frac{\frac{\partial^{3} h}{\partial x_{j_{1}} \partial x_{j_{2}} \partial x_{j_{3}}}}{\sum c_{j} x_{j}+d}+\sum e_{r_{1} r_{2} r_{3}} \frac{\partial \psi^{r_{1}}}{\partial x_{j_{1}}} \frac{\partial \psi^{r_{2}}}{\partial x_{j_{2}}} \frac{\partial \psi^{r_{3}}}{\partial x_{j_{3}}} .
\end{aligned}
$$

Hence letting

we must solve

$$
\left\{\begin{array}{l}
\psi^{1}=x_{1}+h\left(x_{2}, \cdots, x_{n}\right) \\
\psi^{i}=x_{i}
\end{array}\right.
$$

$$
\frac{\partial^{3} h}{\partial x_{j_{1}} \partial x_{j_{2}} \partial x_{j_{3}}}=\frac{1}{2} e_{j_{1} j_{2} j_{3}}
$$

and this is easily done. Similarly the pseudogroup is given by restricting $h$ to polynomials of degree $\leqq 2$.

3. $G^{n}$

As $n$ gets larger the calculations grow more involved; I will merely sketch the situation for these higher groups.

The general $G^{n}$ is built over a $G^{n-1}$; by the equivalence result on this $G^{n-1}$, we can assume $g^{n-1}=\left\langle k^{1}, \cdots, k^{n-1}\right\rangle$. Then by lemma $16, g^{n}$ is determined 
by $\varphi \in \Lambda^{n} k^{n-1} / k^{n}$;

$$
g^{n}=\left\{\left\langle A_{1}, \cdots, A_{n}\right\rangle \mid\left\langle A_{1}, \cdots, A_{n-1}\right\rangle \in g^{n-1} \text { and } A_{n}=-\frac{1}{n-1}\left[d \rho\left(A_{1}\right) \varphi\right]+k^{n}\right\} .
$$

There are four remaining kernel sequences, (2), (7), (18b) and (19b).

Look first at (2); then $\varphi=\left(\begin{array}{cccc}0 & \cdots & 0 & \psi_{j_{1} \cdots j_{n}} \\ 0 & \cdots & 0 & 0\end{array}\right)$. But if $A_{1}=\left(\begin{array}{cc}a & b_{i} \\ 0 & d^{i}{ }_{j}\end{array}\right),\left[A_{1}, \varphi\right]^{1}{ }_{j_{1} \cdots j_{n}}$ is $\sum A^{i}{ }_{k} \varphi_{j_{1} \cdots j_{n}}^{k}-\sum \varphi_{j_{1} \cdots \cdots j_{n}} A_{j_{\lambda}}^{k}=a \psi_{j_{1} \cdots j_{n}}-\sum \phi_{j_{1} \cdots \cdots \cdots j_{n}} d_{j_{\lambda} \cdot}^{k}$. Hence

$$
A^{1}{ }_{j_{1} \cdots j_{n}}=\frac{1}{n-1}\left[\Sigma \psi_{j_{1} \cdots k \cdots j_{n}} d_{j_{\lambda}}^{k}-a \phi_{j_{1} \cdots j_{n}}\right] \text {. }
$$

LEMMA 58.

$$
\begin{aligned}
& G^{n}=\left\{\left(\begin{array}{cc}
a & b_{i} \\
0 & d^{i}{ }_{j}
\end{array}\right),\left(\begin{array}{ccc}
0 & 0 & e_{i j} \\
0 & 0 & 0
\end{array}\right), \cdots,\left(\begin{array}{lllll}
0 & 0 & \cdots & 0 & e_{j_{1} \cdots j_{n-1}} \\
0 & 0 & \cdots & 0 & 0
\end{array}\right),\right. \\
& \left.\left(\begin{array}{cc}
0 \cdots 0 & \frac{1}{n-1}\left[\sum \phi_{k_{1} \cdots k_{n}} d^{k_{1}}{ }_{j_{1}} \cdots d^{k_{n}}{ }_{j_{n}}-a \phi_{j_{1} \cdots j_{n}}\right] \\
0 \cdots 0 & 0
\end{array}\right)\right\} \text {. }
\end{aligned}
$$

ProOF. This clearly has the correct Lie algebra; it suffices if it is a group. But $(\xi \circ \eta)^{1}{ }_{j_{1} \cdots j_{n}}=\xi^{1}{ }_{1} \eta^{1}{ }_{j_{1} \cdots j_{n}}+\sum \xi^{1}{ }_{{ }_{1} \cdots k_{n}} \eta^{k_{1}}{ }_{j_{1}} \cdots \eta^{k_{n}}{ }_{j_{n}}$ in this case, since all other terms in the formula in theorem 1 clearly vanish. Hence if $\xi$ is represented as above, and $\eta$ is the same with $\sim$ over each element, we have

$$
\begin{aligned}
& (\xi \circ \eta)^{1}{ }_{j_{1} \cdots j_{n}}=\frac{1}{n-1}\left[a\left(\sum \phi_{k_{1} \cdots k_{n}} \tilde{d}^{k_{1}}{ }_{j_{1} \cdots}\right]^{k_{n}}{ }_{j_{n}}-\tilde{a} \psi_{j_{1} \cdots j_{n}}\right) \\
& \left.+\Sigma\left(\psi_{k_{1} \cdots k_{n}} d^{k_{1}}{ }_{r_{1}} \cdots d^{k_{n}}{ }_{r_{n}}-a \psi_{r_{1} \cdots r_{n}}\right) d^{r_{1}{ }_{j_{1}}} \ldots d^{r_{n}{ }_{j_{n}}}\right] \\
& =\frac{1}{n-1}\left[\Sigma \phi_{k_{1} \cdots k_{n}}(d \tilde{d})^{k_{1}}{ }_{j_{1}} \cdots(d \tilde{d})^{k_{j_{n}}}-a \tilde{a} \phi_{j_{1} \cdots j_{n}}\right]
\end{aligned}
$$

and so a group.

The resulting pseudogroup for $\varphi$ is

where

$$
\left\{\begin{array}{l}
y_{1}=c x_{1}+g\left(x_{2}, \cdots, x_{n}\right) \\
y_{i}=\Sigma a_{i j} x_{j}+b_{i}
\end{array}\right.
$$

$$
\frac{\partial^{n} g}{\partial x_{j_{1}} \cdots \partial x_{j_{n}}}=\frac{1}{n-1}\left[\Sigma \psi_{k_{1} \cdots k_{n}} a_{k_{1 j_{1}}} \cdots a_{k_{n} j_{n}}-c \psi_{j_{1} \cdots j_{n}}\right] \text {. }
$$

In particular, for $\phi=0$, this is a polynomial of degree $n-1$.

Now choose $h$ with $\frac{\partial^{n} h}{\partial x_{j_{1}} \cdots \partial x_{j_{n}}}=\frac{1}{n-1} \psi_{j_{1} \cdots j_{n}}$. Consider

$$
\vartheta=\left\{\begin{array}{l}
y_{1}=x_{1}+h\left(x_{2}, \cdots, x_{n}\right) \\
y_{i}=x_{i} .
\end{array}\right.
$$


Then for any $f$ in the above pseudogroup of the form

we have

$$
f=\left\{\begin{array}{l}
y_{1}=c x_{1}+h\left(x_{2}, \cdots, x_{n}\right) \\
y_{i}=\sum a_{i j} x_{j}+b_{i}
\end{array}\right.
$$

$$
\vartheta^{-1} \circ f \circ \vartheta=\left\{\begin{array}{l}
y_{1}=c x_{1}+c h\left(x_{2}, \cdots, x_{n}\right)+g\left(x_{2}, \cdots, x_{n}\right)-h\left(\sum a_{i j} x_{j}+b_{i}\right) \\
y_{i}=\Sigma a_{i j} x_{j}+b_{i} .
\end{array}\right.
$$

I claim this is in the pseudogroup for $\psi=0 \Leftrightarrow f$ is in the original pseudogroup. Indeed,

$$
\begin{aligned}
& \frac{\partial^{n}}{\partial x_{j_{1}} \cdots \partial x_{j_{n}}}\left[\operatorname{ch}\left(x_{2}, \cdots, x_{n}\right)+g\left(x_{2}, \cdots, x_{n}\right)-h\left(\sum a_{i j} x_{j}+b_{i}\right)\right] \\
& \quad=\frac{1}{n-1} c \psi_{j_{1} \cdots j_{n}}+\frac{\partial^{n} g}{\partial x_{j_{1}} \cdots \partial x_{j_{n}}}-\frac{1}{n-1} \sum \psi_{k_{1} \cdots k_{n}} a_{k_{1} j_{1}} \cdots a_{k_{n} j_{n}}
\end{aligned}
$$

so this is zero if and only if

$$
\frac{\partial^{n} g}{\partial x_{j_{1}} \cdots \partial x_{j_{n}}}=\frac{1}{n-1}\left[\Sigma \psi_{k_{1} \cdots k_{n}} a_{k_{1} j_{1}} \cdots a_{k_{n} j_{n}}-c \psi_{j_{1} \cdots j_{n}}\right] \text {. }
$$

This solves the equivalence problem and yields $2_{n}$ in the list of pseudogroups.

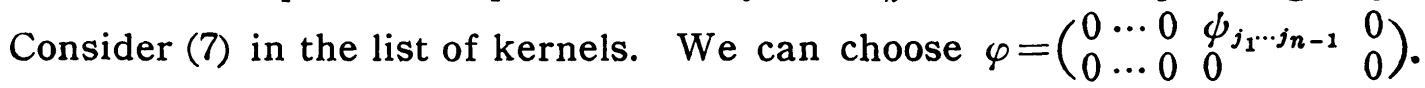
LEMMA 59.

$$
\begin{aligned}
& g^{n}=\left\{\left(\begin{array}{cc}
a & b_{i} \\
0 & d^{i}{ }_{j}
\end{array}\right),\left(\begin{array}{ccc}
0 & e_{i} & e_{i j} \\
& & \\
0 & 0 & 0
\end{array}\right), \cdots,\left(\begin{array}{cccc}
0 & \cdots & 0 & e_{j_{1} \cdots j_{n-1}} e_{j_{1} \cdots j_{n}} \\
& & 0 & 0 \\
0 & \cdots & 0 & 0
\end{array}\right),\right. \\
& \left.\left(\begin{array}{ccc}
0 \cdots 0 & \frac{1}{n-1} \sum \psi_{j_{1} \cdots \cdots j_{n-1}} d_{j_{\lambda}}^{k} & e_{j_{1} \cdots j_{n}} \\
0 \cdots 0 & 0 & 0
\end{array}\right)\right\}
\end{aligned}
$$

Proof. A simple calculation.

We now sketch the construction of $G^{n}$. If $e_{j_{1} \cdots j_{n-1}}$ were arbitrary, the i pseudogroup would be

$$
\left\{\begin{array}{l}
y_{1}=x_{1} g\left(x_{2}, \cdots, x_{n}\right)+h\left(x_{2}, \cdots, x_{n}\right) \\
y_{i}=\Sigma a_{i j} x_{j}+b_{i}
\end{array}\right.
$$

I claim that if $\phi=0$, the pseudogroup is as above with the restriction $g\left(x_{2}, \cdots, x_{n}\right)=e^{P_{n-1}\left(x_{2}, \cdots, x_{n}\right)}, P_{n-1}$ a polynomial of degree $\leqq n-1$. Let us use this to construct $G^{n}$. Since $g \neq 0$, we can write $g\left(x_{2}, \cdots, x_{n}\right)=e^{l\left(x_{2}, \cdots, x_{n}\right)}$. Then

$$
\frac{\partial^{n} y_{1}}{\partial x_{j_{1}} \cdots \partial x_{j_{n-1}} \partial x_{1}}=\frac{\partial^{n-1}}{\partial x_{j_{1}} \cdots \partial x_{j_{n-1}}} e^{i\left(x_{2}, \cdots, x_{n}\right)}
$$




$$
\begin{aligned}
= & e^{l\left(x_{2}, \cdots, x_{n}\right)} \frac{\partial^{n-1} l}{\partial x_{j_{1}} \cdots \partial x_{j_{n-1}}} \\
& + \text { terms of lower order in } l .
\end{aligned}
$$

These terms of lower order in $l$ can in turn be expressed in terms of $\frac{\partial^{n-k} y_{1}}{\partial x_{1} \partial x_{j_{1}} \cdots \partial x_{j_{n-k-1}}}$. Hence $e_{1 j_{1} \cdots j_{n-1}}=e^{l} \frac{\partial^{n-1} l}{\partial x_{j_{1}} \cdots \partial x_{j_{n-1}}}+$ expression in $e_{j_{1} \cdots j_{k}}$ $(k<n-1)$. Call this last expression $\sigma$. Then let

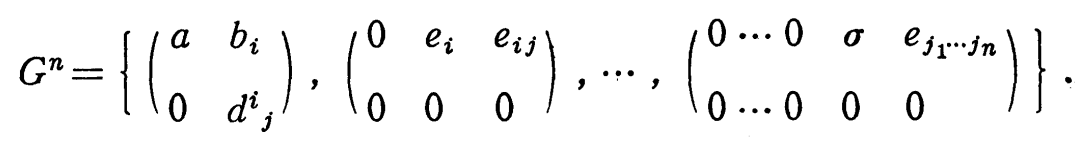

This would produce the above pseudogroup, as $e_{1 j_{1} \cdots j_{n-1}}=\sigma$ implies $\frac{\partial^{n-1} l}{\partial x_{j_{1}} \cdots \partial x_{j_{n-1}}}$ $=0$.

Now presumably we must show that this is a group with the correct algebra; these calculations I skip.

Suppose we let $\psi$ be arbitrary.

LEMMA 60.

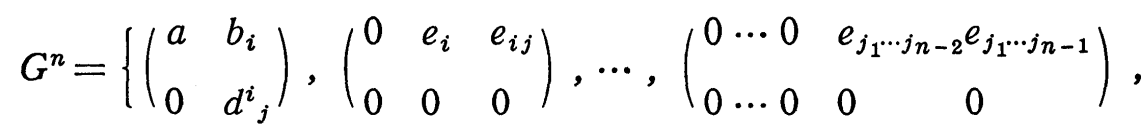

$$
\begin{aligned}
& \left.\left(\begin{array}{ccc}
0 \cdots 0 & \sigma+\frac{1}{n-1}\left[a \sum \phi_{k_{1} \cdots k_{n-1}} d_{j_{1}}^{k_{1}} \cdots d^{k_{n-1} j_{n-1}}-a \psi_{j_{1} \cdots j_{n-1}}\right. & e_{j_{1} \cdots j_{n}} \\
0 \cdots 0 & 0 & 0
\end{array}\right)\right\} \text {. }
\end{aligned}
$$

Proof. This clearly has the correct algebra (assuming $G^{n}$ for $\phi=0$ does).

But if $\xi$ is as above, $\eta$ the same with $\sim$ over each element, a short calculation shows

$$
\begin{aligned}
& (\xi \circ \eta)^{1}{ }_{1 j_{1} \cdots j_{n-1}}=(\text { composition of these two for } \psi=0) \\
& +\frac{1}{n-1} \Sigma\left[a \tilde{a} \psi_{k_{1} \cdots k_{n-1}} d^{k_{j_{1}}} \ldots d^{k_{n-1}{ }_{j_{n-1}}}\right. \\
& +a \tilde{a} \psi_{k_{1} \cdots k_{n}} d^{k_{1}{ }_{l_{1}}} \cdots d^{k_{n-1}{ }_{l_{n-1}}} d_{j_{j_{1}}} \ldots d^{l_{n-1}{ }_{j_{n-1}}} \\
& -a \tilde{a} \psi_{j_{1} \cdots j_{n-1}}-\tilde{a} a \psi_{k_{1} \cdots k_{n-1}} \tilde{d}^{k_{j_{1}}} \ldots d^{\left.k_{n-1}{ }_{j_{n-1}}\right]}
\end{aligned}
$$

and thus $G^{n}$ is a group. The resulting pseudogroup is then

where

$$
\left\{\begin{array}{l}
y_{1}=x_{1} e^{l\left(x_{2}, \cdots, x_{n}\right)}+h\left(x_{2}, \cdots, x_{n}\right) \\
y_{i}=\Sigma a_{i j} x_{j}+b_{i}
\end{array}\right.
$$

or

$$
e^{l} \frac{\partial^{n-1} l}{\partial x_{j_{1}} \cdots \partial x_{j_{n-1}}}=\frac{1}{n-1} e^{l} \sum \phi_{k_{1} \cdots k_{n-1}} a_{j_{1}}^{k_{1}} \cdots a^{k_{n-1} j_{n-1}}
$$




$$
-\frac{\partial^{n-1} l}{\partial x_{j_{1}} \cdots \partial x_{j_{n-1}}}=\frac{1}{n-1} \sum \phi_{k_{1} \cdots k_{n-1}} a_{j_{1}}^{k_{1}} \cdots a^{k_{n-1} j_{n-1}} .
$$

Now pick $R\left(x_{2}, \cdots, x_{n}\right), \frac{\partial^{n-1} R}{\partial x_{j_{1}} \cdots \partial x_{j_{n-1}}}=\frac{1}{n-1} \psi_{j_{1} \cdots j_{n-1}}$. Consider

Then if

$$
\vartheta=\left\{\begin{array}{l}
y_{1}=x_{1} e^{R\left(x_{2}, \cdots, x_{n}\right)} \\
y_{i}=x_{i}
\end{array}\right.
$$

$$
\begin{aligned}
f & =\left\{\begin{array}{l}
y_{1}=x_{1} e^{l\left(x_{2}, \cdots, x_{n}\right)}+h\left(x_{2}, \cdots, x_{n}\right) \\
y_{i}=\sum a_{i j} x_{j}+b_{i}
\end{array}\right. \\
\vartheta^{-1} \circ f \circ \vartheta & =\left\{\begin{array}{l}
y_{1}=x_{1} e^{\left[R\left(x_{2}, \cdots, x_{n}\right)+h\left(x_{2}, \cdots, x_{n}\right)-R\left(\Sigma a_{i j} x_{j}\right)\right]}+h\left(x_{2}, \cdots, x_{n}\right) e^{-R} \\
y_{i}=\sum a_{i j} x_{j}+b_{i} .
\end{array}\right.
\end{aligned}
$$

I claim $\vartheta^{-1} \circ f \circ \vartheta$ is in the pseudogroup for $\psi=0$ if and only if $f$ is in the original pseudogroup. Indeed

$$
\begin{aligned}
& \frac{\partial^{n-1}(R+h-R)}{\partial x_{j_{1}} \cdots \partial x_{j_{n-1}}} \\
& \quad=\frac{1}{n-1} \psi_{j_{1} \cdots j_{n-1}}+\frac{\partial^{n-1} h}{\partial x_{j_{1}} \cdots \partial x_{j_{n-1}}}-\frac{1}{n-1} \sum \phi_{k_{1} \cdots k_{n-1}} a_{j_{j_{1}} \cdots a^{k_{n-1}}{ }_{j_{n-1}}}
\end{aligned}
$$

and the result is clear. We get $(6 n)$ on the list of pseudogroups.

Consider finally (18b) and (19b). Then $\varphi=\left(\begin{array}{llll}0 & \cdots & 0 & \phi_{j_{1} \cdots j_{n}} \\ 0 & \cdots & 0 & 0\end{array}\right)$ and

$$
\begin{aligned}
& g^{n}=\left\{\left(\begin{array}{ll}
a & b_{i} \\
0 & d^{i}{ }_{j}
\end{array}\right),\left(\begin{array}{ccc}
0 & \frac{n-1}{2} d^{i}{ }_{i i} & e_{i j} \\
0 & 0 & \Omega\left(T^{2}\right)
\end{array}\right),\left(\begin{array}{llll}
0 & 0 & 0 & e_{i j k} \\
0 & 0 & 0 & 0
\end{array}\right),\right. \\
& \left.\cdots,\left(\begin{array}{cc}
0 \cdots 0 & \frac{1}{n-1}\left(\sum \phi_{j_{1} \cdots \cdots j_{n}} d_{j_{\lambda}}^{k}-a \phi_{j_{1} \cdot j_{n}}\right) \\
0 \cdots 0 & 0
\end{array}\right)\right\} .
\end{aligned}
$$

The pseudogroup, if this last term were arbitrary, is

$$
\left\{\begin{array}{l}
y_{1}=\frac{A x_{1}+h\left(x_{2}, \cdots, x_{n}\right)}{\left(\sum c_{j} x_{j}+d\right)^{n-1}} \\
y_{i}=\frac{\sum a_{i j} x_{j}+b_{i}}{\sum c_{j} x_{j}+d} .
\end{array}\right.
$$

I claim that if $\phi=0$, the pseudogroup is defined by $h=P_{n-1}$, a polynomial of degree $\leqq n-1$.

We use the same reasoning here as in the above example; namely 


$$
\frac{\partial^{n} y_{1}}{\partial x_{j_{1}} \cdots \partial x_{j_{n}}}=\frac{\frac{\partial^{n} h}{\partial x_{j_{1}} \cdots \partial x_{j_{n}}}}{\left(\sum c_{j} x_{j}+d\right)^{n-1}}+(\text { terms of lower order in } h)
$$

so calling these terms $\sigma$ we expect the group $G^{n}$ to be the same as the group for the full pseudogroup except that $e_{j_{1} \cdots j_{n}}=\sigma$. (Again, we omit the check that this is correct.)

A calculation similar to those above shows that if $\phi \neq 0$, we must add

$$
\frac{1}{n-1}\left[\Sigma \psi_{k_{1} \cdots k_{n}} d_{j_{1}}^{k_{1}} \cdots d_{j_{n}}^{k_{n}}-a \psi_{j_{1} \cdots j_{n}}\right]
$$

to the $e_{j_{1} \cdots j_{n}}$ term.

Let

Then if

$$
\vartheta=\left\{\begin{array}{l}
y_{1}=x_{1}+k\left(x_{2}, \cdots, x_{n}\right) \\
y_{i}=x_{i}
\end{array}\right.
$$

$$
\begin{aligned}
f=\left\{\begin{aligned}
y_{1} & =\frac{A x_{1}+h\left(x_{2}, \cdots, x_{n}\right)}{\left(\sum c_{j} x_{j}+d\right)^{n-1}} \\
y_{i} & =\frac{\sum a_{i j} x_{j}+b_{i}}{\sum c_{j} x_{j}+d}
\end{aligned}\right. \\
\vartheta^{-1} \circ f \circ \vartheta=\left\{\begin{aligned}
& y_{1}=A x_{1}+A k\left(x_{2}, \cdots, x_{n}\right) \\
&-\frac{\left(\sum c_{j} x_{j}+d\right)^{n-1} k\left(\frac{\sum a_{i j} x_{j}+b_{i}}{\sum c_{k} x_{k}+d}\right)+h\left(x_{2}, \cdots, x_{n}\right)}{\left(\sum c_{j} x_{j}+d\right)^{n-1}} \\
& y_{i}=\frac{\sum a_{i j} x_{j}+b_{i}}{\sum c_{j} x_{j}+d} .
\end{aligned}\right.
\end{aligned}
$$

To solve the equivalence problem, we simply need choose $k$ so that when $\frac{\partial^{n} h}{\partial x_{j_{1}} \cdots \partial x_{j_{n}}}=0$,

$$
\begin{gathered}
\left(\sum c_{j} x_{j}+d\right)^{-(n-1)} \frac{\partial^{n}}{\partial x_{j_{1}} \cdots \partial x_{j_{n}}}\left[A k\left(x_{2}, \cdots, x_{n}\right)+h\left(x_{2}, \cdots, x_{n}\right)\right. \\
\left.-\left(\sum c_{j} x_{j}+d\right)^{n-1} k\left(\frac{\sum a_{i j} x_{j}+b_{i}}{\sum c_{j} x_{j}+d}\right)\right] \\
=\frac{1}{n-1}\left[\sum \phi_{k_{1} \cdots k_{n}} \frac{\partial}{\partial x_{j_{1}}}\left(\frac{\sum a_{k_{1} x_{j}}+b_{k_{1}}}{\sum c_{j} x_{j}+d}\right) \cdots \frac{\partial}{\partial x_{j_{n}}}\left(\frac{\sum a_{k_{n}} x_{j}+b_{k_{n}}}{\sum c_{j} x_{j}+d}\right)\right. \\
\left.-\frac{A}{\left(\sum c_{j} x_{j}+d\right)^{n-1}} \psi_{j_{1} \cdots j_{n}}\right] .
\end{gathered}
$$

If $\frac{\partial^{n} k}{\partial x_{j_{1}} \cdots \partial x_{j_{n}}}=-\frac{1}{n-1} \psi_{j_{1} \cdots j_{n}}$, we are done provided:

LEMMA 61. Given an arbitrary $C^{\infty}$ function $k\left(x_{1}, \cdots, x_{n}\right)$, 


$$
\begin{aligned}
& \frac{\partial^{n}}{\frac{\partial x_{j_{1}} \cdots \partial x_{j n}}{\left(\sum c_{j} x_{j}+d\right)^{n-1}}}\left[\left(\sum c_{j} x_{j}+d\right)^{n-1} k\left(\frac{\sum a_{i j} x_{j}+b_{i}}{\sum c_{j} x_{j}+d}\right)\right] \\
& \quad=\left.\frac{\partial^{n} k}{\partial x_{k_{1}} \cdots \partial x_{k_{n}}}\right|_{\left(\frac{\Sigma a_{i j} x_{j}+b_{i}}{\Sigma c_{j} x_{j}+d}\right)} \frac{\partial}{\partial x_{j_{1}}}\left(\frac{\sum a_{k_{1}} x_{j}+b_{k_{1}}}{\sum c_{j} x_{j}+d}\right) \cdots \frac{\partial}{\partial x_{j_{n}}}\left(\frac{\sum a_{k_{n}} x_{j}+b_{k_{n}}}{\sum c_{j} x_{j}+d}\right) .
\end{aligned}
$$

PROOF. The lemma is obvious if $k$ is a polynomial $P_{n-1}$ of degree $\leqq n-1$. It is also obvious (at a fixed point $p$ ) if $\frac{\partial k}{\partial x_{j}}(p), \cdots, \frac{\partial^{n-1} k}{\partial x_{j_{1}} \cdots \partial x_{j_{n-1}}}(p)$ are all zero. But any $\psi$ may be written $\psi=P_{n-1}+\psi_{0}$ where $\psi_{0}$ has this property at $p$; consequently it is true of any $\phi$ at $p$, and as $p$ is arbitrary, true in general.

\section{University of Oregon}

\section{BIBLIOGRAPHY}

[1] S. Bochner and R.C. Gunning, Infinite linear pseudogroups of transformations, Ann. of Math., 75 (1962), 93-104.

[2] H. Boerner, Representations of groups, North-Holland Publishing Co., Amsterdam, 1963.

[3] V. Guillemin and S. Sternberg, An algebraic model of transitive differential geometry, Bull. Amer. Math. Soc., 70 (1964), 16-47.

[4] R.C. Gunning, Complex analytic pseudogroups and the quasi-uniformization of complex manifolds (unpublished).

[5] R. C. Gunning, Connections for a class of pseudogroup structures, in Proceedings of the conference on complex analysis (Minneapolis), Springer-Verlag, New York, 1965.

[6] S. Helgason, Differential geometry and symmetric spaces, Academic Press, N. Y., 1962.

[7] S. Kobayashi and T. Nagano, On filtered Lie algebras and geometric structures I, II, III, J. Math. and Mech., 13 (1964), 875-908; 14 (1965), 513-521, 679-706.

[8] R.M. Koch, Pseudogroups associated with the one dimensional foliation group (I), J. Math. Soc. Japan, 23 (1971), 149-180.

[9] I. M. Singer and Shlomo Sternberg, The infinite groups of Lie and Cartan, part I (the transitive groups), J. Analyse Math., 15 (1965), 4-113.

[10] H. Weyl, The classical groups, Princeton Univ. Press, Princeton, 1946. 